\title{
ON FINITE DISPLACEMENTS OF CURVED ANNULAR ELASTIC MEMBRANES WITHOUT WRINKLING
}

\author{
BY
}

ARMIN BECK AND HANS GRABMÜLLER

Institut für Angewandte Mathematik, Universität Erlangen-Nürnberg, Erlangen, Germany

\begin{abstract}
Axisymmetric deformations of curved annular elastic membranes subjected to vertical surface loads and radial edge loads or displacements are considered within Reissner's finite-rotation theory of thin shells of revolution, assuming linear stress-strain relations. The principal stresses in the membrane are determined by the solutions of a nonlinear second-order ODE, dependent on a geodesic variable. Analytical methods are used in order to determine the range of those boundary data for which the solutions of the differential equation are wrinkle-free in the sense that both the radial and the circumferential stress components are nonnegative everywhere.
\end{abstract}

1. Introduction. For many events, a linear theory of elastic membranes and thin shells of revolution is not applicable, for instance, if the applied load is not small enough. In these cases a nonlinear theory is required. Of particular interest are geometrically nonlinear theories where nonlinear strain-displacement relations but a linear stress-strain relation like Hooke's law are assumed.

A membrane theory of this kind that is widely applied and that covers the special case of aximembranes, originates from E. Reissner's theory of thin shells of revolution having negligible bending stiffness (the finite-rotation theory [16]). Aximembranes are membranes of revolution under axisymmetric loads, and a geometrical configuration of great significance in applications is the curved annular aximembrane, which will be the subject of the present paper.

Actually, here we shall discuss axisymmetric deformations of annular membranes subjected to vertical surface loads and to several uniform edge loads such as radial stresses and radial displacements within the framework of finite-rotation theory. We are especially interested in the determination of those boundary parameters that allow for a stable equilibrium only, in the sense that both the principal stresses are nonnegative everywhere in the membrane. A stable equilibrium prevents the membrane from buckling. The solutions corresponding to a stable membrane state will be called wrinkle-free since buckling of a stretched membrane sometimes is termed "wrinkling" (see, e.g., D. Steigman [19]).

Received January 4, 1993.

1991 Mathematics Subject Classification. Primary 73C05, 73K10, 73H99, 34B15.

(C) 1995 Brown University 
The mathematical problems of existence and uniqueness of wrinkle-free solutions for curved circular membranes under variable vertical loads have been solved only recently by A. Beck and H. Grabmüller [3], but the corresponding case of curved annular aximembranes has apparently not been investigated as yet. The present results originate from the doctoral thesis of the first author [2] with a number of considerable improvements obtained by the use of a suitable transformation of variables. Some work on curved annular aximembranes was also done by J. Arango [1]. In his thesis he studied rotationally symmetric deformations of aximembranes under uniform normal pressure. His analysis was governed by a shooting method, which was introduced in membrane theory by A. J. Callegari and E. L. Reiss [4].

In our work a quite different method is employed. The existence of solutions of the stress boundary-value problem is proved by an integral equation technique utilizing Schauder's fixed-point theorem, while positivity and uniqueness are obtained from a generalized maximum principle. Concerning the displacement boundaryvalue problems, a mapping argument is used, which was first proposed by R. Pirner [14] and which has been successfully applied later in a refined version in order to study stable equilibria of flat annular membranes, not only within a simplified version of Reissner's finite-rotation theory [7, 10,11,21] but also within the so-called smallfinite-deflection theory [7, 8, 9, 12]. We refer to [22] for a review. The idea of this method consists of mapping the set of boundary parameters related to a stable state of the stress problem into the parameter sets of the displacement problems.

Our work proceeds as follows. The basic equations of finite-rotation theory for aximembranes are supplied in Sec. 2, following the representation of R. A. Clark and O. S. Narayanaswamy [5]. Using a composition of a geodesic transformation and a transformation due to E. Schwerin [17] the stress and displacement problems occur in the form of a nonlinear second-order ODE boundary-value problem. After having introduced the notation of regular tensile solutions, we determine in Sec. 3 the nonnegative solutions of the stress problem. The boundary-value problems involving displacement data are examined in Sec. 4 employing the mapping argument. Here the sets of physically admissible boundary data are subdivided into complementary domains of existence and nonexistence of tensile solutions. In Sec. 5 we finally discuss the totality of boundary parameters related to wrinkle-free solutions of the stress and displacement problems.

It should be emphasized that the flat annular membrane is incorporated in our analysis as a special case, $p=$ const (see Sec. 2), and that our considerations are not restricted to the simplified Reissner model. So the results of the present paper significantly extend previous work on flat annular aximembranes.

2. Formulation of the problem. In three-dimensional space, the undeformed membrane is generated by a rotating meridian curve whose parametric representation is taken in cylindrical coordinates $(r, \theta, \zeta)$ in the form $r=r(\sigma), \zeta=\zeta(\sigma)$. The parameter $\sigma$ denotes the arclength along a meridian, and we assume that $0 \leq \sigma \leq$ $L<\infty$. If $\Phi$ denotes the angle of inclination measured from the radial axis to the meridional tangent of the deformed membrane, and if $S_{\Phi}$ and $S_{\theta}$ are pseudo-stress resultants that measure the tension in the deformed membrane per unit length of the 
undeformed membrane, the state of the deformed membrane is completely described by the quantities

$$
M=r S_{\Phi} \cos \Phi, \quad N=r S_{\Phi} \sin \Phi, \quad H=r S_{\theta} ;
$$

see R. A. Clark and O. S. Narayanaswamy [5] for details.

If both stresses $S_{\Phi}$ and $S_{\theta}$ are nonnegative, the deformed membrane is called stably equilibrated in the sense of D. Steigmann [19]. Rotations have to be restricted to $0 \leq|\Phi(\sigma)| \leq \frac{\pi}{2}, 0 \leq \sigma \leq L$. Therefore, wrinkling cannot occur in any parts of the membrane, if both the radial and circumferential stresses $M$ and $H$ are nonnegative everywhere.

We assume that a vertical surface load $q_{\zeta}(\sigma)$ is distributed per unit undeformed area. According to [5], the basic equations for the unknowns $M, N$, and $H$ can now be written in the form

$$
\begin{aligned}
r \frac{d}{d \sigma} M & =H, \\
r \frac{d}{d \sigma} N & =-r^{2} q_{\zeta}, \\
r \frac{d}{d \sigma} H & =M+E h r\left(\frac{M}{\sqrt{M^{2}+N^{2}}}-\frac{d}{d \sigma} r\right)-\nu r^{2} q_{\zeta} \frac{N}{\sqrt{M^{2}+N^{2}}} .
\end{aligned}
$$

Here $E>0$ denotes Young's modulus of elasticity, $\nu$ Poisson's ratio (restricted to $0 \leq \nu \leq 0.5)$ and $h>0$ the thickness of the membrane.

The generating meridian should have smoothness $r \in C^{1}[0, L]$. Assuming an inner radius $0<a=r(0)$ of the annulus and an outer radius $b=r(L)>a$, simple geometrical considerations lead to the requirements that $a \leq r(\sigma) \leq a+\sigma$, $\sigma \in[0, L]$, and $0<r^{\prime}(\sigma) \leq 1, \sigma \in[0, L)$. In view of this it becomes obvious that the geodesic transformation

$$
\sigma \mapsto \xi:=\kappa(\sigma), \quad \kappa(\sigma):=\exp \left(-\int_{\sigma}^{L} \frac{d \tau}{r(\tau)}\right), \quad \sigma \in[0, L],
$$

establishes a $C^{2}$-diffeomorphism from the segment $[0, L]$ onto $[\varepsilon, 1]$, letting $\varepsilon:=$ $\kappa(0)>0$. For further simplification we shall use E. Schwerin's transformation [17]

$$
\xi \mapsto t:=\frac{\xi^{2}-\varepsilon^{2}}{\omega}, \quad \xi \in[\varepsilon, 1], \quad \omega:=1-\varepsilon^{2},
$$

which maps the segment $[\varepsilon, 1]$ homeomorphically onto $[0,1]$.

In the sequel, $I_{0}$ denotes the segment $0<t<1$, and $I$ its closure. Generally, the load function $q_{\zeta}$ should be bounded such that $q_{0}:=\max \left|q_{\zeta}(\sigma)\right|>0$ exists. Letting $\bar{q}(t):=q_{\zeta}(\sigma) / q_{0}, k(t):=\sqrt{\omega t+\varepsilon^{2}}, k(t) p(t):=\omega r(\sigma) / L, x(t) / k(t):=$ $M(\sigma) /\left(L^{2} q_{0}\right), y(t):=N(\sigma) /\left(L^{2} q_{0}\right), C:=E h /\left(4 L q_{0}\right)$, and eliminating the quantity $H$ in (2.2), one obtains

$$
\begin{aligned}
x^{\prime \prime}(t) & =F(t, x(t)), \quad t \in I_{0}, \\
y^{\prime}(t) & =-\frac{1}{2 \omega} p^{2}(t) \bar{q}(t), \quad t \in I_{0},
\end{aligned}
$$


where primes are used to indicate derivatives with respect to $t$ and where

$$
\begin{aligned}
F(t, x) & :=\frac{C \omega p(t)}{k^{2}(t)}\left(\frac{x}{\sqrt{x^{2}+\Theta^{2}(t)}}-1\right)-2 C p^{\prime}(t)-\frac{\nu p^{2}(t) \bar{q}(t)}{4 k(t)} \frac{\Theta(t)}{\sqrt{x^{2}+\Theta^{2}(t)}}, \\
\Theta(t) & :=k(t) y(t) .
\end{aligned}
$$

An integration of Eq. (2.6) yields

$$
y(t)=y_{0}-\frac{1}{2 \omega} \int_{0}^{t} p^{2}(\tau) \bar{q}(\tau) d \tau=y_{1}+\frac{1}{2 \omega} \int_{t}^{1} p^{2}(\tau) \bar{q}(\tau) d \tau, \quad t \in I .
$$

Here and in the sequel it is always assumed that one of the boundary conditions associated with the system (2.2) has the form $y(0)=y_{0}$ or $y(1)=y_{1}$. Thus, $F(t, x)$ can be considered as a function depending on the known variable $t \in I$ and a single unknown variable $x$. Neglecting the $\nu$-term in (2.7), we get the so-called simplified Reissner model (cf. $[13,18]$ ), which has been the basis of extensive investigations on flat annular and circular membranes.

Notice that the function $p(t)$ has smoothness $p \in C^{1}(I)$ and that

$$
p^{\prime}(t)=\frac{\omega^{2}}{2 L k^{3}(t)} r(\sigma)\left(r^{\prime}(\sigma)-1\right) \leq 0, \quad t \in I .
$$

Therefore, $p$ is bounded by $0<p(1)=b \omega / L \leq p(t) \leq p(0)=a \omega /(L \varepsilon)$. For a flat membrane, $p(t)=1+\frac{a}{b}=1+\varepsilon$ and $p^{\prime}(t) \equiv 0$.

The following assumption $(Q)$ is needed to prevent $y(t)$ from vanishing at an inner point $t \in I_{0}$ and should be valid throughout the whole paper:

The load function $0 \leq \bar{q} \in C(I)$ satisfies $y^{2}(t)>0, t \in I_{0}$, and $y_{j}^{2}+\bar{q}(j)>0$, $j=0,1$.

If the deformed membrane is stably equilibrated, that is, if in particular $S_{\Phi} \geq 0$ holds, then the sign of the quantity $r S_{\Phi} \sin \Phi=L^{2} q_{0} y$ determines the curvature of the membrane. A convex membrane state is characterized by $y \geq 0$ (this requires a sufficiently large $y_{0}$ ), while a concave membrane state is characterized by $y \leq 0$.

The radial displacement of a membrane point after deformation is given by [5]

$$
\tilde{u}=\frac{1}{E h}\left(r \frac{d}{d \sigma} M-\nu \sqrt{M^{2}+N^{2}}\right) .
$$

Letting $u(t) / k(t):=(2 \omega C / L) \tilde{u}(\sigma)$, we obtain

$$
u(t)=k^{2}(t) x^{\prime}(t)-\frac{\omega}{2}\left(x(t)+\nu \sqrt{x^{2}(t)+\Theta^{2}(t)}\right), \quad t \in I .
$$

Now, a set of four different boundary-value problems (BVP) is obtained when Eq. (2.5) is associated with the boundary conditions

$$
\begin{aligned}
& x(0)=s \quad \text { or } \quad B_{0}[x]:=\varepsilon^{2} x^{\prime}(0)-\frac{\omega}{2}\left(x(0)+\nu \sqrt{x^{2}(0)+\varepsilon^{2} y_{0}^{2}}\right)=h, \\
& x(1)=S \quad \text { or } \quad B_{1}[x]:=x^{\prime}(1)-\frac{\omega}{2}\left(x(1)+\nu \sqrt{x^{2}(1)+y_{1}^{2}}\right)=H .
\end{aligned}
$$


Physically, this means prescribing either the radial stresses or the radial displacements at the inner and outer edges of the annulus.

Subsequently, reference will be made to the above problems as the BVPs $(s, S)$, $(h, S),(s, H)$, or $(h, H)$ with obvious notation. It should be emphasized that the relation $\cos \Phi=x / \sqrt{x^{2}+\Theta^{2}}$ permits other classes of boundary conditions. In any case, these conditions can be reduced to (2.10) or (2.11), if two of the three quantities $x(j), y(j), \Phi(j)$ are prescribed for $j=0$ and $j=1$, respectively.

In this paper, only nonnegative solutions $x(t)$ are of interest (the so-called tensile solutions). It is useful to classify them according to regular tensile solutions ( $\mathrm{rt}-$ solutions for short), i.e., functions $x \in C^{2}\left(I_{0}\right) \cap C^{1}(I)$ satisfying $x(t)>0$ for $t \in I$, and regular nonnegative solutions (rn-solutions) with the same smoothness of an rtsolution but with a less strict positivity $x(t)>0, t \in I_{0}$.

3. Tensile solutions under Dirichlet data. In what follows, $E$ denotes the Banach space $C(I)$ of real-valued continuous functions over $I$, equipped with the usual max-norm $\|\cdot\|$. Using Green's function, one shows by a standard technique that an rn-solution $x(t)$ of the BVP $(s, S)$ admits an integral representation

$$
x(t)=f(t)-\int_{0}^{1} K(t, \tau) F(\tau, x(\tau)) d \tau, \quad t \in I,
$$

where $f(t):=S t+s(1-t)$ and where the kernel function

$$
K(t, \tau):= \begin{cases}(1-t) \tau, & 0 \leq \tau \leq t \leq 1 \\ (1-\tau) t, & 0 \leq t \leq \tau \leq 1\end{cases}
$$

is nonnegative and bounded above by 1 . Conversely, any solution $x \in E$ of the integral equation (3.1) possesses the required regularity $x \in C^{2}\left(I_{0}\right) \cap C^{1}(I)$ and solves the BVP $(s, S)$ being, however, not necessarily positive. Therefore, rn-solutions constitute a subset of the solution variety $x \in E$ of Eq. (3.1). In order to supply a result on existence of a continuous solution of (3.1), we employ a fixed-point argument. For this purpose, let $s \geq 0$ and $S \geq 0$ be fixed. We introduce a nonlinear operator $T: E \rightarrow E$ and a convex set $M_{s, S} \subset E$ by setting

$$
[T x](t):=f(t)-\int_{0}^{1} K(t, \tau) F(\tau, x(\tau)) d \tau, \quad x \in E, t \in I,
$$

$$
M_{s, S}:=\left\{x \in E\left|\exists H \in C\left(I_{0}\right):\right| H(t) \mid \leq K_{1} \text { and } x(t):=f(t)-\int_{0}^{1} K(t, \tau) H(\tau) d \tau\right\},
$$

where $K_{1}:=\left\|2 C\left(\omega p / k^{2}-p^{\prime}\right)+\nu p^{2} \bar{q} /(4 k)\right\|<\infty$. Using arguments as in [3, Lemma 3.1], the operator $T$ is shown to fulfill all requirements of the Schauder fixed-point theorem (see, e.g., $[20, \S 7, \mathrm{XII}]$ ) when acting on the closure of the set $M_{s, S}$. Since $T(E) \subset M_{s, S}$, there must exist at least one fixed-point $x=T x \in$ $M_{s, S}$ of the required regularity of an rn-solution. Furthermore, if we let $\widetilde{F}(t):=$ $\omega C p(t) /\left(k^{2}(t) \sqrt{x^{2}(t)+\Theta^{2}(t)}\right) \geq 0$ and if $y \geq 0$ is assumed, it follows from condition $(Q)$ that $F(t, x(t))-\widetilde{F}(t) x(t) \leq 0$ holds for all $t \in I_{0}$. Thus $x^{\prime \prime}(t)-\widetilde{F}(t) x(t) \leq$ 0 , and the maximum principle of M. H. Protter and H. F. Weinberger (see [15, Thm. 
3]) applies to $-x$ with the result that $x$ cannot attain a local minimum $x\left(t_{0}\right) \leq 0$ at an interior point $t_{0} \in I_{0}$. Therefore, $x(t)$ must be an rn-solution of the BVP $(s, S)$.

If $y<0$ holds, solutions $x \in E$ of Eq. (3.1) are by no means positive, not even for $s>0$ and $S>0$. In order to guarantee positivity, we need a somewhat sharper condition for $\bar{q}(t)$, namely:

In addition to $(Q)$ the load function satisfies

$$
\bar{q}(t) \operatorname{sign}(-y(t)) \leq \frac{4 C L}{\nu} \frac{d}{d \sigma} \ln r(\sigma), \quad t \in I_{0} .
$$

Observe that condition (Q1) is void, if $y \geq 0$ holds or if the simplified Reissner model is considered. In any other cases, numerical computations show that solutions $x(t)$ of the BVP $(s, S)$ fail to be nonnegative everywhere, if $s \geq 0$ and $S \geq 0$ are small numbers and if condition (Q1) is violated. However, since

$$
\frac{4 C L}{\nu} \frac{d}{d \sigma} \ln r(\sigma)=\frac{4 C}{\nu p^{2}(t)}\left(\frac{\omega p(t)}{k(t)}+2 p^{\prime}(t) k(t)\right), \quad t \in I,
$$

a straightforward calculation again yields $F(t, x(t))-\widetilde{F}(t) x(t) \leq 0, t \in I_{0}$, provided that condition $(\mathrm{Q} 1)$ holds. As before, the maximum principle is applicable and we obtain the following main result on existence of rn-solutions to the BVP $(s, S)$.

TheOREM 3.1. Suppose condition (Q1) holds. Then, for each fixed $s \geq 0, S \geq 0$, there exists at least one rn-solution $x(t)$ of the BVP $(s, S)$. For $s>0, S>0, x(t)$ is an $r$-solution.

The problem of uniqueness of rn-solutions is easily solved if $y \geq 0$ occurs or if the simplified Reissner model is considered. Indeed, let $F(t, x)$ for fixed $t \in I_{0}$ be interpreted as a function of $x \geq 0$. The quantity

$$
D(x):=\frac{d}{d x} F(t, x)=\frac{p(t)}{4 k^{2}(t)} \frac{4 \omega C \Theta^{2}(t)+\nu k(t) p(t) \bar{q}(t) \Theta(t) x}{\left(x^{2}+\Theta^{2}(t)\right)^{3 / 2}}
$$

is nonnegative for $x \geq 0$. Suppose there exist two rn-solutions $x_{1}$ and $x_{2}$ of the BVP $(s, S)$. Letting $d(t):=x_{1}(t)-x_{2}(t), t \in I$, one has $d(0)=0=d(1)$ as well as

$$
d^{\prime \prime}(t)=D\left(x_{\mu}(t)\right) d(t), \quad t \in I_{0},
$$

where the intermediate variable $x_{\mu} \geq 0$ is defined by $x_{\mu}(t):=x_{1}(t)+\mu(t)\left(x_{2}(t)-\right.$ $\left.x_{1}(t)\right), 0<\mu(t)<1$. Thus, Eq. (3.5) can be subjected to the maximum principle (see [15, Thm. 3]), from which we obtain $d \equiv 0$, and therefore $x_{1}=x_{2}$.

In general, if $y<0$ is assumed, $D(x)$ may become negative for some values of $x>0$. Since $D(0) \geq 0$ and since $D(x) \rightarrow 0$ as $x \rightarrow+\infty$, a calculation of the zeros of $D^{\prime}(x)$ proves that a global negative minimum is attained at

$$
x_{0}=\frac{|\Theta|}{4}\left(3 \lambda^{-1}+\sqrt{9 \lambda^{-2}+8}\right),
$$


where we have used the notation $\lambda=\lambda(t):=\nu k(t) p(t) \bar{q}(t) /(4 \omega C) \geq 0$. If the continuous and monotone decreasing function $g(\lambda)$ is introduced by

$$
g(\lambda):=\frac{\sqrt{6}}{9} \frac{\sqrt{9+8 \lambda^{2}}-3}{\lambda^{2}\left(3+4 \lambda^{2}+\sqrt{9+8 \lambda^{2}}\right)^{1 / 2}},
$$

it follows that $0 \leq g(\lambda) \leq g(0)=4 / 27$ and $g(\lambda) \rightarrow 0$ as $\lambda \rightarrow+\infty$, while

$$
D\left(x_{0}\right)=-\frac{\omega C p(t) \lambda^{3}(t)}{|\Theta(t)| k^{2}(t)} g(\lambda(t)) \leq 0, \quad t \in I_{0}
$$

(see also [3]). Now, Eq. (3.5) is no longer subordinate to the maximum principle and we do not know a general solution of the uniqueness problem. However, a partial solution can be derived from the generalized maximum principle of Protter and Weinberger [15] provided there exists a function $w \in C^{2}(I), w>0$, such that $h(t):=D\left(x_{\mu}(t)\right)-w^{\prime \prime}(t) / w(t) \geq 0$ holds for all $t \in I_{0}$. In this case, if a new dependent variable $v:=d / w$ is introduced, Eq. (3.5) has to be replaced by

$$
w(t) v^{\prime \prime}+2 w^{\prime}(t) v^{\prime}=h(t) w(t) v, \quad t \in I_{0} .
$$

This equation can again be subjected to the maximum principle, from which we obtain $v \equiv 0$, and therefore $x_{1}=x_{2}$.

In order to determine a sufficient condition for the crucial hypothesis $h(t) \geq 0$, we found it most appropriate to consider the gauge function

$$
w(t):=\frac{\sin (\alpha t+\beta)}{\sin \beta}, \quad t \in I,
$$

to which the following condition for the uniqueness of rn-solutions is related:

If $D(x) \geq 0$ holds for all $x \geq 0$ then let $\alpha=0$. Otherwise assume there exist two numbers $\alpha>0, \beta>0$ with $0<\alpha+\beta<\pi$, such that for all $t \in I_{0}$

$$
\alpha^{2}-\frac{\omega C p(t) \lambda^{3}(t)}{|\Theta(t)| k^{2}(t)} g(\lambda(t)) \geq 0 .
$$

Now, the result on uniqueness can be stated as follows.

TheOrem 3.2. Suppose condition $\left(\mathrm{Q}_{\alpha, \beta}\right)$ holds and let $s \geq 0, S \geq 0$ be fixed. Then there exists at most one rn-solution of the BVP $(s, S)$.

REMARKs. (a) It should be noticed that the preceding theorem does not make any use of the assertion (Q1). Furthermore, up to this point the condition $y_{j}^{2}+\bar{q}(j)>0$, $j=0,1$, has not influenced any parts of the existence and uniqueness results and may be cancelled from $(\mathrm{Q})$. However, it becomes important in later considerations on monotonicity. (b) Condition $\left(\mathrm{Q}_{\alpha, \beta}\right)$ with $\alpha=0$ holds if $y \geq 0$ is assumed or if the simplified Reissner model is considered. In any other cases it depends decisively on the magnitude of $y_{0}$. It should be realized that it is optimal to choose the parameter $\beta>0$ as small as possible. (c) Condition $\left(\mathrm{Q}_{\alpha, \beta}\right)$ may be replaced by the stronger condition

$$
\alpha^{2}-\frac{4}{27} \frac{\omega C p(t) \lambda^{3}(t)}{|\Theta(t)| k^{2}(t)} \geq 0, \quad t \in I_{0},
$$


which is independent of $g(\lambda)$ and thus easier to check. (d) If in addition (Q1) is satisfied, then it follows that $\lambda(t) \leq 1$. While $\lambda^{2} g(\lambda)$ is monotone increasing for $\lambda \geq 0$, condition $\left(\mathrm{Q}_{\alpha, \beta}\right)$ may also be replaced by

$$
\alpha^{2}-\frac{\omega C p(t) \lambda(t)}{|\Theta(t)| k^{2}(t)} g(1) \geq 0, \quad t \in I_{0}, \quad\left(\mathrm{Q}_{\alpha, \beta}^{\prime \prime}\right)
$$

where $g(1) \doteq 0.0917$.

4. Tensile solutions involving displacement data. Throughout this section it is always assumed that condition (Q1) holds. Theorem 3.1 shows that the quarter plane $\operatorname{Ex}(s, S):=\{(s, S) \mid s \geq 0, S \geq 0\}$ represents the domain of existence of rnsolutions to the $\operatorname{BVP}(s, S)$. If, in addition, condition $\left(\mathrm{Q}_{\alpha, \beta}\right)$ is valid, then any point $(s, S) \in E x(s, S)$ is uniquely related to an rn-solution $x(t)$ and its derivative $x^{\prime}(t)$. Denoting these relations by $A_{t}(s, S):=x(t)$ and $D A_{t}(s, S):=x^{\prime}(t)$, the displacement boundary conditions

$$
\begin{aligned}
& h=B_{0}[x]=\varepsilon^{2} D A_{0}(s, S)-\frac{\omega}{2}\left(s+\nu \sqrt{s^{2}+\varepsilon^{2} y_{0}^{2}}\right), \\
& H=B_{1}[x]=D A_{1}(s, S)-\frac{\omega}{2}\left(S+\nu \sqrt{S^{2}+y_{1}^{2}}\right)
\end{aligned}
$$

may be interpreted as mappings from the set $E x(s, S)$ onto the set of those boundary data $(h, S),(s, H)$, and $(h, H)$, where rn-solutions of the corresponding BVPs do exist.

4.1. Continuous dependence on Dirichlet data. The main purpose of this section is to show that the mappings $A_{t}$ and $D A_{t}$ depend monotonely and continuously on the boundary data $s \geq 0$ and $S \geq 0$. We begin with a result on monotonicity.

LemMA 4.1. Suppose condition $(Q 1)$ holds. Let $s_{j} \geq 0, S_{j} \geq 0, j=1,2$, be fixed and define $x_{j}(t):=A_{t}\left(s_{j}, S_{j}\right)$ as well as $d(t):=x_{1}(t)-x_{2}(t), t \in I$. Then we have

(a) Monotonicity of the differential equation:

$$
d^{\prime \prime}(t)\left\{\begin{array}{l}
\geq 0 \text { for } d(t) \geq 0, \\
\leq 0 \text { for } d(t) \leq 0,
\end{array} \quad t \in I_{0},\right.
$$

provided that $D(x) \geq 0$ holds for all $x \geq 0$.

(b) Monotonicity of the solutions: If $s_{1} \geq s_{2}$ and $S_{1} \geq S_{2}$ then $d(t) \geq 0$ for all $t \in I$, provided that condition $\left(\mathrm{Q}_{\alpha, \beta}\right)$ holds.

(c) Monotonicity of the derivatives: Suppose condition $\left(\mathrm{Q}_{\alpha, \beta}\right)$ holds within the more restricted range of $0<\alpha+\beta \leq \pi / 2$. (i) If $s_{1}=s_{2}$ and $S_{1}>S_{2}$ then $d^{\prime}(t)>0$ for all $t \in I$. (ii) If $s_{1}>s_{2}$ and $S_{1}=S_{2}$ then $d^{\prime}(t)<0$ for all $t \in I$.

(d) Monotonicity of the displacements: Suppose condition $\left(\mathrm{Q}_{\alpha, \beta}\right)$ holds with $0<$ $\alpha+\beta \leq \pi / 2$. (i) If $s_{1}=s_{2}$ and $S_{1}>S_{2}$ then $B_{0}\left[x_{1}\right]>B_{0}\left[x_{2}\right]$ and $B_{1}\left[x_{1}\right]>B_{1}\left[x_{2}\right]$, where the latter assertion requires the additional condition of

$$
\tan (\alpha+\beta)=\frac{\alpha}{\mu}, \quad \mu:=\frac{\omega}{2}(1+\nu),
$$

if $D(x) \geq 0$ is violated. (ii) If $s_{1}>s_{2}$ and $S_{1}=S_{2}$ then $B_{i}\left[x_{1}\right]<B_{i}\left[x_{2}\right], i=0,1$. 
Proof. Assertion (a) is an immediate consequence of Eq. (3.5). In order to prove assertion (b), let us introduce a new dependent variable $v:=d / w$, with $w(t)$ as defined in (3.8) if $D(x) \geq 0$ is violated. Otherwise let $w \equiv 1$. The function $v(t)$ solves the differential equation (3.7), which may be written in the form

$$
v^{\prime \prime}+\frac{2 w^{\prime}(t)}{w(t)} v^{\prime}-h(t) v=0, \quad t \in I_{0}
$$

Since $v(0) \geq 0$ and $v(1) \geq 0$, the maximum principle applies to (4.4) with the result that $v(t) \geq 0, t \in I$.

Concerning assertion (c), we observe that a transformation $d(t) \mapsto d(1-t)$ reduces case (ii) to case (i). For a proof of (i), let us first show that the function $v(t)$ defined in (b) now satisfies $v^{\prime}(t)>0, t \in I$. Notice that $v(0)=0$ is a minimum. Clearly, the coefficients $2 w^{\prime}(t) / w(t)$ and $-h(t)$ of Eq. (4.4) are bounded on every closed subinterval of $I_{0}$. Moreover, the quantity

$$
\frac{2 w^{\prime}(t)}{w(t)}-t h(t)=\frac{2 w^{\prime}(t)}{w(t)}-t \alpha^{2}-t D\left(x_{\mu}(t)\right)
$$

is bounded at $t=0$. This becomes obvious if $s_{1}>0$ or $y_{0} \neq 0$ holds because it follows from $x_{\mu}^{2}(0)+\Theta^{2}(0)>0$ that

$$
\left|t D\left(x_{\mu}(t)\right)\right| \leq \frac{t p(t)}{4 k^{2}(t)} \frac{4 \omega C+\nu k(t) p(t) \bar{q}(t)}{\sqrt{x_{\mu}^{2}(t)+\Theta^{2}(t)}} \rightarrow 0 \text { as } t \rightarrow 0+.
$$

Likewise, if $s_{1}=0=y_{0}$ is assumed, we have $0 \leq x_{j}^{\prime}(0)=\lim _{t \rightarrow 0+} x_{j}(t) / t$ and therefore, $0 \leq x_{\mu}(t) / t \leq K_{2}$ for some finite constant $K_{2}$. From condition (Q) it follows that $\lim _{t \rightarrow 0+} \Theta(t) / t=-k(0) p^{2}(0) \bar{q}(0) /(2 \omega)<0$. Thus, the boundedness at $t=0$ becomes obvious from the representation

$$
t D\left(x_{\mu}(t)\right)=\frac{p(t)}{4 k^{2}(t)} \frac{4 \omega C \frac{\boldsymbol{\Theta}^{2}(t)}{t^{2}}+\nu k(t) p(t) \bar{q}(t) \frac{\boldsymbol{\Theta}(t)}{t} \frac{x_{\mu}(t)}{t}}{\left(\left(\frac{x_{\mu}(t)}{t}\right)^{2}+\left(\frac{\boldsymbol{\Theta}(t)}{t}\right)^{2}\right)^{3 / 2}} .
$$

Now, Theorem 4 of [15] applies to $-v$ with the result that $v^{\prime}(0)>0$. In constructing a contradiction to the assertion $v^{\prime}(t)>0$, let us assume that there exists a point $0<t_{0} \leq 1$ with $v^{\prime}\left(t_{0}\right)=0$ and $v^{\prime}(t)>0$ for $0 \leq t \leq t_{0}$. Since $\left(w^{2}(t) v^{\prime}\right)^{\prime}=$ $h(t) w^{2}(t) v \geq 0$ holds by (4.4), we have reached a contradiction

$$
0 \leq \int_{0}^{t_{0}}\left(w^{2}(\tau) v^{\prime}(\tau)\right)^{\prime} d \tau=-w^{2}(0) v^{\prime}(0)<0 .
$$

It follows that $v^{\prime}(t)>0, t \in I$, and consequently

$$
d^{\prime}(t)=w^{\prime}(t) v(t)+w(t) v^{\prime}(t) \geq w(t) v^{\prime}(t)>0 .
$$

Case (ii) of assertion (d) is easily obtained from (c) because we have $B_{0}\left[x_{1}\right]-$ $B_{0}\left[x_{2}\right] \leq \varepsilon^{2} d^{\prime}(0)-\frac{\omega}{2} d(0)$ and $B_{1}\left[x_{1}\right]-B_{1}\left[x_{2}\right]=d^{\prime}(1)$. As for a proof of (i), we notice that $B_{0}\left[x_{1}\right]-B_{0}\left[x_{2}\right]=\varepsilon^{2} d^{\prime}(0)>0$ by assertion (c), while $B_{1}\left[x_{1}\right]-B_{1}\left[x_{2}\right] \geq$ 
$d^{\prime}(1)-\mu d(1)$. Using convexity of $d(t)$ as shown in (a), we obtain $d^{\prime}(1) \geq d(1)>0$. Since $\mu$ does not exceed 0.75 , the asserted monotonicity holds in the case of $D(x) \geq$ 0 . Otherwise we utilize condition $\left(Q_{\alpha, \beta}\right)$ and the relation (4.3) in order to obtain

$$
d^{\prime}(1)-\mu d(1)=\left(w^{\prime}(1)-\mu w(1)\right) v(1)+w(1) v^{\prime}(1)=w(1) v^{\prime}(1)>0 .
$$

So the lemma is proved.

The next result concerns strong continuity of the mappings $A_{t}$ and $D A_{t}$ with respect to the boundary data $s \geq 0$ and $S \geq 0$.

Lemma 4.2. The mappings $A_{t}(s, S)$ and $D A_{t}(s, S)$ are strongly continuous at each point $(s, S) \in E x(s, S)$, provided that the assertions of Lemma 4.1(b) and (c) hold.

Proof. Let $\left(s_{j}, S_{j}\right) \in \operatorname{Ex}(s, S)$ be fixed for $j=1,2$. With the previous notation $d(t):=x_{1}(t)-x_{2}(t)$ it follows from Lemma 4.1(b) and (c) that the expression $\Delta \equiv$ $\left\|A_{t}\left(s_{1}, S_{1}\right)-A_{t}\left(s_{2}, S_{2}\right)\right\|$ equals $d(0)=s_{1}-s_{2}$ or $d(1)=S_{1}-S_{2}$, if we specialize to parameters $s_{1}>s_{2}, S_{1}=S_{2}$ or $s_{1}=s_{2}, S_{1}>S_{2}$, respectively. Thus, the general case leads to $\Delta \leq\left|s_{1}-s_{2}\right|+\left|S_{1}-S_{2}\right|$ by virtue of the triangle inequality.

In order to prove continuity of the mapping $D A_{t}(s, S)$ we again specialize to the above parameter régime. Since a transformation $d(t) \mapsto d(1-t)$ reduces the second case to the first one, it suffices to consider $s_{1}>s_{2}$ and $S_{1}=S_{2}$ only. Here we have $\Delta_{12} \equiv\left\|D A_{t}\left(s_{1}, S_{1}\right)-D A_{t}\left(s_{2}, S_{2}\right)\right\|=\left\|d^{\prime}\right\|$ with

$$
d^{\prime}(t)=s_{2}-s_{1}+\int_{0}^{t} \tau D_{F}(\tau) d \tau-\int_{t}^{1}(1-\tau) D_{F}(\tau) d \tau, \quad t \in I,
$$

where the integral representation (3.1) is utilized and where $D_{F}(t):=F\left(t, x_{1}(t)\right)$ $F\left(t, x_{2}(t)\right)$. Using continuity of $A_{t}(s, S)$ as proved before, we obtain $\lim _{s_{1} \rightarrow s_{2}} D_{F}(t)$ $=0$, pointwise for each $t \in I_{0}$. Since $\left|D_{F}(t)\right|$ is dominated by the constant $2 K_{1}$, an application of Lebesgue's dominated convergence theorem to (4.5) yields

$$
\limsup _{s_{1} \rightarrow s_{2}} \Delta_{12} \leq \limsup _{s_{1} \rightarrow s_{2}} \int_{0}^{1}\left|D_{F}(\tau)\right| d \tau=0,
$$

and thus strong continuity is proved.

By virtue of (4.1), (4.2), and Lemma 4.2, the two-parameter manifolds $\beta_{i}(s, S):=$ $B_{i}[x], i=0,1$, are continuous over $\operatorname{Ex}(s, S)$, and so must be any projection $\beta_{i}\left(s_{0}, S\right)$ or $\beta_{i}\left(s, S_{0}\right)$ for a fixed $s_{0} \geq 0$ or $S_{0} \geq 0$. The following properties are important for further investigations where we let

$$
\gamma_{i}(s):=\beta_{i}(s, 0), \quad s \geq 0, \quad \Gamma_{i}(S):=\beta_{i}(0, S), \quad S \geq 0, \quad i=0,1,
$$

and $K_{0, \infty}:=2 \varepsilon^{2} C(P-p(0)), K_{1, \infty}:=2 C(P-p(1)), P:=\int_{0}^{1} p(\tau) d \tau$.

Lemma 4.3. Suppose conditions $(\mathrm{Q} 1)$ and $\left(\mathrm{Q}_{\alpha, \beta}\right)$ hold with $0<\alpha+\beta \leq \pi / 2$. Then we have the following.

(a) The continuous projections $\beta_{i}\left(\cdot, S_{0}\right): \overline{\mathbb{R}}_{+} \rightarrow \mathbb{R}, S_{0} \geq 0$, are strictly decreasing, and so must be $\gamma_{i}$. The range of $\beta_{i}\left(\cdot, S_{0}\right)$ is the segment $\left(-\infty, \Gamma_{i}\left(S_{0}\right)\right]$, and an 
asymptotic form of $\gamma_{i}$ is provided by

$$
\left.\begin{array}{r}
\gamma_{0}(s)+\left(\varepsilon^{2}+\mu\right) s-K_{0, \infty}=o(1) \\
\gamma_{1}(s)+s+\frac{\omega}{2} \nu\left|y_{1}\right|-K_{1, \infty}=o(1)
\end{array}\right\} \quad \text { as } s \rightarrow+\infty .
$$

(b) The continuous projections $\beta_{i}\left(s_{0}, \cdot\right): \overline{\mathbb{R}}_{+} \rightarrow \mathbb{R}, s_{0} \geq 0$, are strictly increasing, and so must be $\Gamma_{i}$. The range of $\beta_{i}\left(s_{0}, \cdot\right)$ is the segment $\left[\gamma_{i}\left(s_{0}\right),+\infty\right)$, and an asymptotic form of $\Gamma_{i}$ is provided by

$$
\left.\begin{array}{rl}
\Gamma_{0}(S)-\varepsilon^{2} S+\frac{\omega}{2} \nu \varepsilon\left|y_{0}\right|-K_{0, \infty} & =o(1) \\
\Gamma_{1}(S)-(1-\mu) S-K_{1, \infty} & =o(1)
\end{array}\right\} \quad \text { as } S \rightarrow+\infty .
$$

If $D(x) \geq 0$ is violated, the assumptions must additionally be restricted by (4.3) for assuring that $\beta_{1}\left(s_{0}, \cdot\right)$ is strictly increasing.

Proof. In view of Lemma 4.1 and Lemma 4.2 nothing is needed except for the asymptotic forms (4.7) and (4.8). A proof of them utilizes the integral representation (3.1) and

$$
x^{\prime}(t)=S-s-\int_{0}^{1} \partial_{t} K(t, \tau) F(\tau, x(\tau)) d \tau, \quad t \in I,
$$

from which it follows that

$$
\begin{aligned}
& \beta_{0}(s, S)=\varepsilon^{2} S-\left(1-\frac{\omega}{2}\right) s-\varepsilon^{2} \int_{0}^{1}(1-\tau) F(\tau, x(\tau)) d \tau-\frac{\omega}{2} \nu \sqrt{s^{2}+\varepsilon^{2} y_{0}^{2}}, \\
& \beta_{1}(s, S)=\left(1-\frac{\omega}{2}\right) S-s+\int_{0}^{1} \tau F(\tau, x(\tau)) d \tau-\frac{\omega}{2} \nu \sqrt{S^{2}+y_{1}^{2}} .
\end{aligned}
$$

Since $|F(t, x)|$ is uniformly bounded with respect to $x$, we obtain from (3.1) and (2.7) that $x(t)=A_{t}(s, S) \rightarrow+\infty$ and $F(t, x(t)) \rightarrow-2 C p^{\prime}(t)$, pointwise for each $t \in I_{0}$ as $s \rightarrow+\infty$ or as $S \rightarrow+\infty$. An application of Lebesgue's dominated convergence theorem to (4.10) leads to the asserted asymptotics.

4.2. Mixed stress and displacement data. In this section we shall prove a result that supplies existence and nonexistence of rt-solutions of the BVPs $(h, S)$ and $(s, H)$ in complementary subdomains of the respective parameter ranges. The idea is to identify the sets

$$
\begin{aligned}
& \operatorname{Ex}(h, S):=\left\{(h, S) \mid h \leq \Gamma_{0}(S), S \geq 0\right\}, \\
& \operatorname{Ex}(s, H):=\left\{(s, H) \mid s \geq 0, H \geq \gamma_{1}(s)\right\}
\end{aligned}
$$

as the homeomorphic images of the set $E x(s, S)$ subjected to the mappings $\left(\beta_{0}, S\right)$ and $\left(s, \beta_{1}\right)$, respectively. Indeed, any fixed $(s, S) \in \operatorname{Ex}(s, S)$ is uniquely related to an rn-solution $x(t)$ to which there corresponds, by Lemma 4.3, a displacement $h:=\beta_{0}(s, S) \leq \Gamma_{0}(S)$ at the inner edge and a displacement $H:=\beta_{1}(s, S) \geq \gamma_{1}(s)$ at the outer edge of the annulus. Thus, the sets (4.11) must enclose all boundary data of possible rn-solutions. Conversely, given a fixed $(h, S) \in E x(h, S)$, then $h$ belongs to the range of $\beta_{0}(\cdot, S)$. Since this mapping is one-to-one, a unique $s \geq 0$ exists such that $h=\beta_{0}(s, S)$. Consequently, the unique rn-solution $x(t)$ related to the point $(s, S) \in \operatorname{Ex}(s, S)$ induces the displacement $h$. It is equally simple to show that a displacement $h>\Gamma_{0}(S)$ does not belong to any rn-solution, while a 
displacement $h=\Gamma_{0}(S)$ is related to the rn-solution $x_{0}(t)$ of the BVP $(s, S), s=0$, and thus $x_{0}(0)=0$ holds. In summary, we have

Theorem 4.4. Suppose conditions $(\mathrm{Q} 1)$ and $\left(\mathrm{Q}_{\alpha, \beta}\right)$ hold with $0<\alpha+\beta \leq \pi / 2$. Let $x_{0}(t):=A_{t}(0, S)$ and define

$$
\Gamma_{0}(S):=\varepsilon^{2} x_{0}^{\prime}(0)-\frac{\omega}{2} \nu \varepsilon\left|y_{0}\right|, \quad S \geq 0 .
$$

Then the strictly increasing and continuous curve $\Gamma_{0}$ subdivides the parameter range $(h, S)$ into complementary subsets of existence and nonexistence as follows.

(a) The BVP $(h, S)$ has a unique rt-solution $x(t)$ if and only if $h<\Gamma_{0}(S)$ and $S>0$.

(b) The BVP $(h, S), h=\Gamma_{0}(S), S \geq 0$, has a unique rn-solution $x(t)$ that coincides with the function $x_{0}(t)$ above and thus satisfies $x(0)=0$ and $x(1)=S$. Finally, the BVP $(h, S), h<h_{0}:=\Gamma_{0}(0), S=0$, has a unique rn-solution $x(t)$ that satisfies $x(0)>0$ and $x(1)=0$.

The BVP $(s, H)$ can be treated in a similar way. Here we have the following

Theorem 4.5. Suppose conditions $(\mathrm{Q} 1)$ and $\left(\mathrm{Q}_{\alpha, \beta}\right)$ hold, with $0<\alpha+\beta \leq \pi / 2$ being additionally restricted by $(4.3)$, if $D(x) \geq 0$ is violated. Let $x_{1}(t):=A_{t}(s, 0)$ and define

$$
\gamma_{1}(s):=x_{1}^{\prime}(1)-\frac{\omega}{2} \nu\left|y_{1}\right|, \quad s \geq 0
$$

Then the strictly decreasing and continuous curve $\gamma_{1}$ subdivides the parameter range $(s, H)$ into complementary subsets of existence and nonexistence as follows.

(a) The $\operatorname{BVP}(s, H)$ has a unique rt-solution $x(t)$ if and only if $s>0$ and $H>\gamma_{1}(s)$.

(b) The BVP $(s, H), s \geq 0, H=\gamma_{1}(s)$, has a unique rn-solution $x(t)$ that coincides with the function $x_{1}(t)$ above and thus satisfies $x(0)=s$ and $x(1)=0$. Finally, the BVP $(s, H), s=0, H>H_{0}:=\gamma_{1}(0)$, has a unique rn-solution $x(t)$ that satisfies $x(0)=0$ and $x(1)>0$.

4.3. The displacement problem. The finite part of the boundary of $\operatorname{Ex}(s, S)$ is mapped by $\left(\beta_{0}, \beta_{1}\right)$ onto a one-parameter manifold $\Sigma=\Sigma_{1} \cup \Sigma_{2} \subset \mathbb{R}^{2}$ consisting of the continuous arcs $\Sigma_{1}:=\left\{(h, H) \mid h=\gamma_{0}(s), H=\gamma_{1}(s), s \geq 0\right\}$ and $\Sigma_{2}:=$ $\left\{(h, H) \mid h=\Gamma_{0}(S), H=\Gamma_{1}(S), S \geq 0\right\}$. Since $\gamma_{i}(0)=\Gamma_{i}(0)$ holds for $i=0,1$, the set $\Sigma$ must be connected.

By construction, any point $(h, H) \in \Sigma$ is related to a unique rn-solution $x(t)$ of the BVP $(h, H)$ with the properties $x(1)=0$ for $(h, H) \in \Sigma_{1}$ and $x(0)=0$ for $(h, H) \in \Sigma_{2}$. Thus, $\Sigma$ should be expected to mark the boundary of the existence domain $\operatorname{Ex}(h, H)$ of rn-solutions of the BVP $(h, H)$. For a proof, we consider annular membranes of restricted aperture only. This precisely means a restriction of the parameter $\varepsilon$ to the range $0<\varepsilon<\sqrt{3} / 3$. In a preliminary step, we eliminate the parameters $s$ and $S$ in the representations of the $\operatorname{arcs} \Sigma_{j}$. Let

$$
h_{0}:=\beta_{0}(0,0)=\gamma_{0}(0)=\Gamma_{0}(0), \quad H_{0}:=\beta_{1}(0,0)=\gamma_{1}(0)=\Gamma_{1}(0),
$$

and define the homeomorphisms $\rho_{1}:\left(-\infty, h_{0}\right] \rightarrow\left(-\infty, H_{0}\right]$ and $\rho_{2}:\left[h_{0},+\infty\right) \rightarrow$ 
$\left[H_{0},+\infty\right)$ by

$$
\rho_{1}(h):=\gamma_{1}\left[\gamma_{0}^{-1}(h)\right], \quad h \leq h_{0}, \quad \rho_{2}(h):=\Gamma_{1}\left[\Gamma_{0}^{-1}(h)\right], \quad h \geq h_{0} .
$$

This allows $\Sigma$ to be written in an explicit form as

$$
\Sigma=\left\{(h, H) \mid H=\rho_{1}(h) \text { for } h \leq h_{0} \text { and } H=\rho_{2}(h) \text { for } h>h_{0}\right\} .
$$

The set $\Sigma$ represents a continuous strictly increasing curve that subdivides the parameter range $(h, H)$ into two simply connected unbounded subsets. As we will show, one of these subsets is the asserted domain of existence of rn-solutions, namely the set

$$
\operatorname{Ex}(h, H):=\left\{(h, H) \mid H \geq \rho_{1}(h) \text { for } h \leq h_{0} \text { and } H \geq \rho_{2}(h) \text { for } h>h_{0}\right\} .
$$

We precede the proof by a result on implicit functions.

In view of Lemma 4.3, the ranges of the mappings $s \mapsto \beta_{0}(s, S), S \geq 0$ fixed, and $S \mapsto \beta_{0}(s, S), s \geq 0$ fixed, enclose the segments $\left(-\infty, h_{0}\right]$ and $\left[h_{0},+\infty\right)$, respectively. Observing the strong monotonicity and continuity of these mappings, it follows from a standard result on implicit functions (cf. [6, Thm. 51]), that there exist uniquely defined continuous functions $\sigma_{h, i}: \overline{\mathbb{R}}_{+} \rightarrow \overline{\mathbb{R}}_{+}, i=0,1$, such that for each fixed $h \in \mathbb{R}$

$$
\beta_{0}\left[\sigma_{h, 0}(S), S\right]=h \leq h_{0}, S \geq 0 \quad \text { and } \quad \beta_{0}\left[s, \sigma_{h, 1}(s)\right]=h \geq h_{0}, s \geq 0 .
$$

The following properties are essential.

LEMMA 4.6. Suppose conditions $(\mathrm{Q} 1)$ and $\left(\mathrm{Q}_{\alpha, \beta}\right)$ hold with $0<\alpha+\beta \leq \pi / 2$. Then we have the following.

(a) The continuous mappings $\sigma_{h, i}, i=0,1$, are strictly increasing.

(b) The continuous mappings $S \mapsto \beta_{1}\left[\sigma_{h, 0}(S), S\right], h \leq h_{0}$, and $s \mapsto \beta_{1}\left[s, \sigma_{h, 1}(s)\right]$, $h \geq h_{0}$, are strictly increasing, provided that $\alpha, \beta$ are additionally restricted by (4.3) and

if $D(x) \geq 0$ is violated.

$$
\tan \beta=\frac{\alpha}{\omega} 2 \varepsilon^{2}
$$

(c) $\beta_{1}\left[\sigma_{h, 0}(0), 0\right]=\rho_{1}(h), h \leq h_{0}$, and $\beta_{1}\left[0, \sigma_{h, 1}(0)\right]=\rho_{2}(h), h \geq h_{0}$.

(d) $\lim _{S \rightarrow+\infty} \beta_{1}\left[\sigma_{h, 0}(S), S\right]=+\infty=\lim _{s \rightarrow+\infty} \beta_{1}\left[s, \sigma_{h, 1}(s)\right]$.

Proof. It suffices to restrict consideration to the case $h \leq h_{0}$, since the case $h>h_{0}$ can be treated analogously. Thus, let $h \leq h_{0}$ be fixed.

(a) Strong monotonicity can be shown as follows. Let $S_{1}>S_{2} \geq 0$ be fixed and define $s_{j}:=\sigma_{h, 0}\left(S_{j}\right), j=1,2$. Using previous notation we have, by definition

$0=\beta_{0}\left(s_{1}, S_{1}\right)-\beta_{0}\left(s_{2}, S_{2}\right)=\varepsilon^{2} d^{\prime}(0)-\frac{\omega}{2}\left(d(0)+\nu\left(\sqrt{s_{1}^{2}+\Theta^{2}(0)}-\sqrt{s_{2}^{2}+\Theta^{2}(0)}\right)\right)$,

and thus the case $s_{1}=s_{2}$ is not permitted by Lemma 4.1(b) and (c). Likewise, if $s_{1}<s_{2}$ is assumed, then $d^{\prime}(0)<0$ holds by (4.19). We let $v(t):=d(t) /(w(1-t))$ with $w \equiv 1$ if $D(x) \geq 0$ holds and with $w$ defined by (3.8) otherwise. While $v(0)<0, v(1)>0$ and

$$
d^{\prime}(0)=-w^{\prime}(1) v(0)+w(1) v^{\prime}(0)<0
$$


it follows that $v^{\prime}(0)<0$. Therefore, a local minimum $v\left(t_{0}\right)<0$ must exist at an inner point $t_{0} \in I_{0}$. By (4.4) we have $v^{\prime \prime}\left(t_{0}\right)=h\left(t_{0}\right) v\left(t_{0}\right) \leq 0$. Since this contradicts the maximum principle it occurs that only $s_{1}>s_{2}$ is possible.

(b) For fixed $S_{1}>S_{2} \geq 0$, let

$$
\Delta \equiv \beta_{1}\left(s_{1}, S_{1}\right)-\beta_{1}\left(s_{2}, S_{2}\right)=B_{1}\left[x_{1}\right]-B_{1}\left[x_{2}\right] \geq d^{\prime}(1)-\mu d(1) .
$$

As was proved in (a), we have $s_{1}>s_{2}$, and therefore $d(t)>0, t \in I$. Utilizing (4.19), it follows that

$$
d^{\prime}(0)-\frac{\omega}{2 \varepsilon^{2}} d(0) \geq 0
$$

where the inequality is strict for $\nu>0$. If $D(x) \geq 0$ holds, then $d(t)$ is convex by Lemma 4.1(a). Since this implies $d^{\prime}(1) \geq d(1)-d(0) \geq d^{\prime}(0)$, we obtain from (4.20) by setting $\kappa:=\omega\left[1-\frac{1}{2}\left(1+\varepsilon^{2}\right)(1+\nu)\right] /\left(2 \varepsilon^{2}\right)$ and observing that $0<\varepsilon<\sqrt{3} / 3$ :

$$
\Delta \geq(1-\mu) d^{\prime}(0)-\mu d(0) \geq \kappa d(0)>0 .
$$

Notice that the preceding analysis covers the special case $\nu=0$. The convexity argument does not apply if $D(x) \geq 0$ is violated. In this case we assume $\nu>0$ and consider $v(t):=d(t) / w(t)$ with $w$ as defined in (3.8). As in the proof of Lemma 4.1(b), we obtain $v(t) \geq 0, t \in I$. Moreover, from (4.18) and (4.20) it follows that

$$
d^{\prime}(0)-\frac{\omega}{2 \varepsilon^{2}} d(0)=w(0) v^{\prime}(0)>0 .
$$

Since $v(t)$ cannot attain a positive local maximum at an interior point $t \in I_{0}$, it follows from $v(0)>0$ and $v^{\prime}(0)>0$ that $v^{\prime}(t)>0$ holds for all $t \in I$. Using (4.3), we have the result

$$
\Delta \geq d^{\prime}(1)-\mu d(1)=w(1) v^{\prime}(1)>0 .
$$

(c) In view of $h=\beta_{0}\left[\sigma_{h, 0}(0), 0\right]=\gamma_{0}\left[\sigma_{h, 0}(0)\right]$, we have $\sigma_{h, 0}(0)=\gamma_{0}^{-1}(h)$, and the asserted relation is a consequence of (4.15).

(d) Since $h=\beta_{0}\left[\sigma_{h, 0}(S), S\right]$, it follows from the representation (4.10a) that $\lim _{S \rightarrow+\infty} \sigma_{h, 0}(S)=+\infty$ with an asymptotic growth like $\sigma_{h, 0}(S)=\varepsilon^{2} S /\left(\varepsilon^{2}+\mu\right)+O(S)$ as $S \rightarrow+\infty$. This, together with the representation (4.10b), yields

$$
\beta_{1}\left[\sigma_{h, 0}(S), S\right]=\frac{\mu \omega(1-\nu)}{2\left(\varepsilon^{2}+\mu\right)} S+O(S) \rightarrow+\infty \quad \text { as } S \rightarrow+\infty .
$$

So the lemma is proved.

REMARK. In order to verify the assumptions of the preceding lemma it must be shown that the relations (4.3) and (4.18) are simultaneously solvable for $\alpha>0$ and $\beta>0$ within the range of $0<\alpha+\beta \leq \pi / 2$. Using inverses of the tan-function, (4.3) and (4.18) may be written as

$$
\alpha+\beta=\arctan \left(\frac{\alpha}{\mu}\right), \quad \beta=\arctan \left(\frac{\alpha \varepsilon^{2}(1+\nu)}{\mu}\right) .
$$

An elimination of the quantity $\beta$ now yields

$$
\alpha=\arctan \left(\frac{\mu \alpha(2 \mu-\nu)}{\mu^{2}+\alpha^{2}(1+\nu-2 \mu)}\right),
$$


and a unique fixed point $\alpha \in(0, \pi / 2)$ exists as long as $2 \mu-\nu>0$ holds. Since this is true when $\varepsilon$ is restricted by $0<\varepsilon<\sqrt{6} / 3$, there exists a unique $\beta>0$ defined by (4.22) within the required range. A table of values of $\alpha$ and $\beta$ dependent on the parameters $\varepsilon$ and $\nu$ is given in Table 1 on p. 542.

Now we are prepared to prove the main result of this section.

Theorem 4.7. Suppose conditions $(\mathrm{Q} 1)$ and $\left(\mathrm{Q}_{\alpha, \beta}\right)$ hold, with $0<\alpha+\beta \leq \pi / 2$ being additionally restricted by (4.3) and (4.18) if $D(x) \geq 0$ is violated. Let $\Gamma_{0}(S)$ and $\gamma_{1}(s)$ be defined as in (4.12) and (4.13), respectively. In addition, define

$$
\begin{aligned}
& \gamma_{0}(s):=\varepsilon^{2} x_{1}^{\prime}(0)-\frac{\omega}{2}\left(s+\nu \sqrt{s^{2}+\varepsilon^{2} y_{0}^{2}}\right), \quad s \geq 0, \\
& \Gamma_{1}(S):=x_{0}^{\prime}(1)-\frac{\omega}{2}\left(s+\nu \sqrt{S^{2}+y_{1}^{2}}\right), \quad S \geq 0,
\end{aligned}
$$

with $x_{0}(t):=A_{t}(0, S)$ and $x_{1}(t):=A_{t}(s, 0)$. Then the set (4.16) represents a continuous one-parameter manifold $\Sigma$ that subdivides the parameter range $(h, H)$ into complementary subsets of existence and nonexistence as follows.

(a) The BVP $(h, H)$ has a unique rt-solution $x(t)$ if and only if

$$
H> \begin{cases}\rho_{1}(h):=\gamma_{1}\left[\gamma_{0}^{-1}(h)\right], & h \leq h_{0}:=\gamma_{0}(0), \\ \rho_{2}(h):=\Gamma_{1}\left[\Gamma_{0}^{-1}(h)\right], & h>h_{0} .\end{cases}
$$

(b) The BVP $(h, H), h \leq h_{0}, H=\rho_{1}(h)$, has a unique rn-solution $x(t)$ that coincides with the function $x_{1}(t)$ above and thus satisfies $x(0)=s=\gamma_{0}^{-1}(h) \geq 0$ and $x(1)=0$. Finally, the BVP $(h, H), h>h_{0}, H=\rho_{2}(h)$, has a unique rn-solution $x(t)$ that coincides with the function $x_{0}(t)$ above and thus satisfies $x(0)=0$ and $x(1)=S=\Gamma_{0}^{-1}(h)>0$.

Proof. It suffices to verify that the set $E x(h, H)$ defined by (4.17) is the homeomorphic image of the set $E x(s, S)$ under the mapping $\left(\beta_{0}, \beta_{1}\right)$. In a first step the image of $\operatorname{Ex}(s, S)$ is shown to be a subset of $\operatorname{Ex}(h, H)$. Indeed, the two semi-axes $s \geq 0, S=0$ and $s=0, S \geq 0$ are mapped onto $\Sigma_{1}, h \leq h_{0}$ and $\Sigma_{2}, h \geq h_{0}$, respectively. For $s>0, S>0$, we let $x(t)$ denote the rt-solution of the corresponding BVP $(s, S)$. If $h:=B_{0}[x]$ satisfies $h \leq h_{0}$, we define $H:=\beta_{1}\left[\sigma_{h, 0}(S), S\right]>\rho_{1}(h)$, and thus $(h, H) \in \operatorname{Ex}(h, H)$. If $h>h_{0}$, the same conclusion holds for $H:=\beta_{1}\left[s, \sigma_{h, 1}(s)\right]>\rho_{2}(h)$.

Next assume that $(h, H) \in \operatorname{Ex}(h, H)$. If $h \leq h_{0}$ holds then we have $H \geq \rho_{1}(h)$. While the range of the mapping $S \mapsto \beta_{1}\left[\sigma_{h, 0}(S), S\right]$ is the segment $\left[\rho_{1}(h),+\infty\right)$, there exists a unique $S \geq 0$ that satisfies $H=\beta_{1}\left[\sigma_{h, 0}(S), S\right]$. With $s:=\sigma_{h, 0}(S) \geq$ 0 , we obtain $(s, S) \in E x(s, S)$. Finally, if $h>h_{0}$ holds, an analogous conclusion can be drawn from $H \geq \rho_{2}(h)$.

5. Tensile solutions without wrinkling. Tensile solutions $x(t)$ as determined in the preceding sections characterize a membrane state with a nonnegative radial stress resultant $M(\sigma)=L^{2} q_{0} x(t) / k(t)$. In this section we shall discuss the more restricted subset of those tensile solutions that are associated with a nonnegative circumferential stress resultant $S_{\theta}$, or equivalently, that characterize the state of a stably equilibrated 
ค โ 0 잉

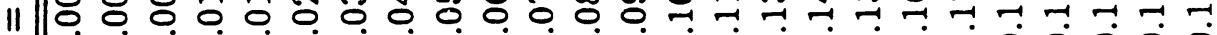

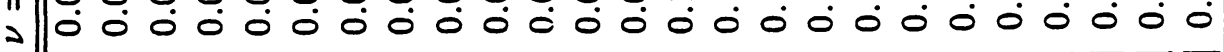

ก 잉

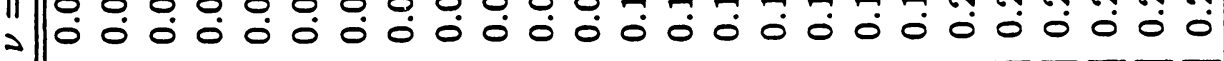

๓ฺ

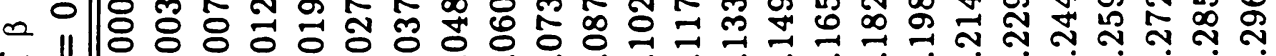
4 د d) శ0 0 ल 11 잉 $80^{-1}$ 잉

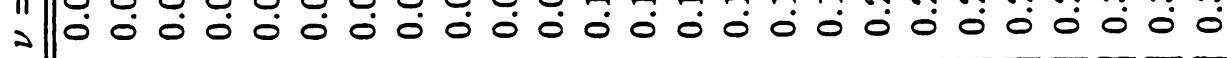

ت.

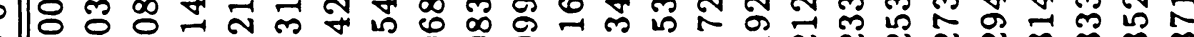

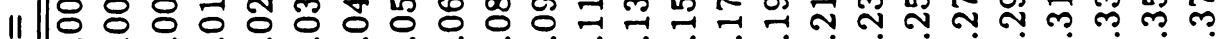

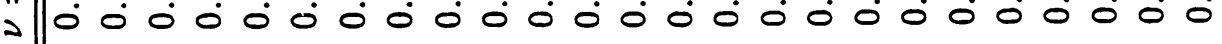

-

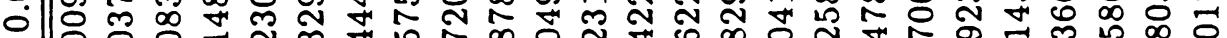

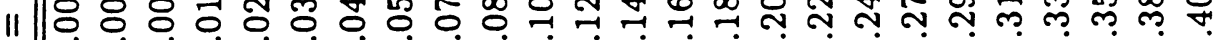
>

n

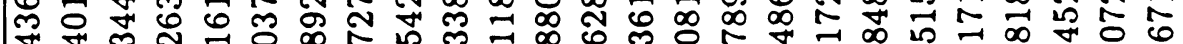

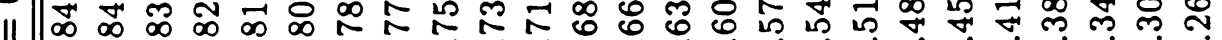

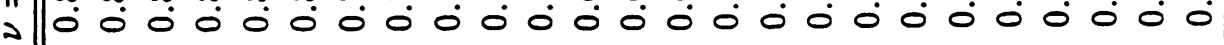

サ.

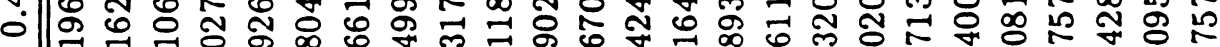

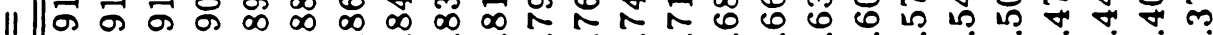

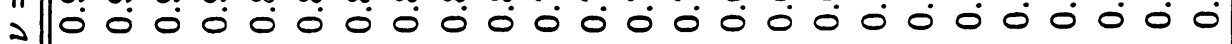

m

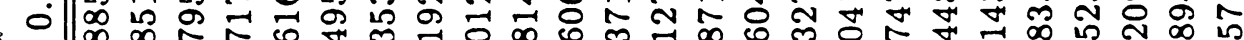

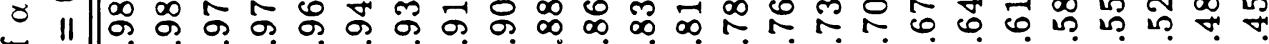
- د

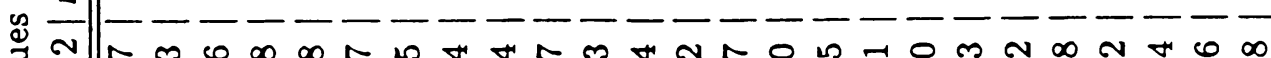
미 ᄀ

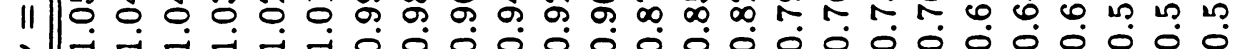

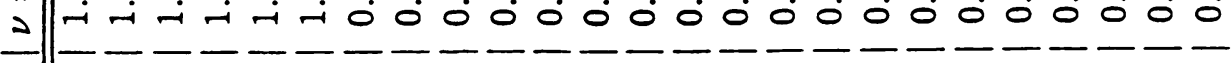

-1) 어 0 \%

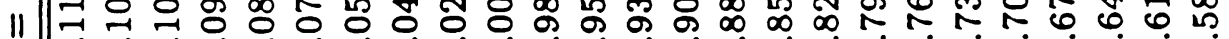

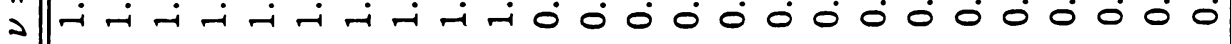

ה 0 m

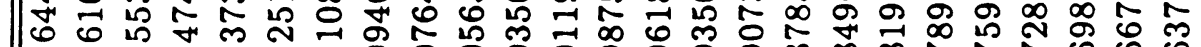
$\|$ II

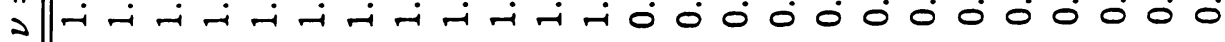
강

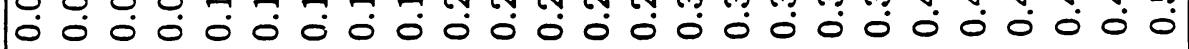


membrane. In view of (2.1), (2.2a), (2.3), and (2.4), we have

$$
S_{\theta}(\sigma)=\frac{d}{d \sigma} M(\sigma)=\frac{c_{1}}{p(t)} \frac{d}{d t}\left(\frac{x(t)}{\sqrt{t+\gamma}}\right) \exp \left(-\int_{\sigma}^{L} \frac{d \tau}{r(\tau)}\right), \quad t \in I,
$$

where $\gamma:=\varepsilon^{2} / \omega>0$ and $c_{1}:=2 L q_{0} / \sqrt{\omega}>0$. Since the function

$$
\rho(t):=\frac{p(t)}{c_{1}} \exp \left(\int_{\sigma}^{L} \frac{d \tau}{r(\tau)}\right) S_{\theta}(\sigma)=\frac{d}{d t}\left(\frac{x(t)}{\sqrt{t+\gamma}}\right), \quad t \in I,
$$

preserves both the regularity and positivity of $S_{\theta}$, an examination of $S_{\theta}$ may be restricted to the discussion of $\rho(t)$ alone.

5.1. Wrinkle-free solutions of the stress problem. The dependence of rn-solutions $x(t):=A_{t}(s, S)$ on Dirichlet data passes over to $\rho(t)$ and will be expressed by writing $\rho(t)$ in the form

$$
\rho(t)=\rho_{t}(s, S)=\frac{2(t+\gamma) x^{\prime}(t)-x(t)}{2(t+\gamma)^{3 / 2}}, \quad t \in I, s \geq 0, S \geq 0 .
$$

In a first step we discuss monotonicity with respect to $s$ and $S$. Using previous notation, we consider

$$
D_{\rho}(t):=\rho_{t}\left(s_{1}, S_{1}\right)-\rho_{t}\left(s_{2}, S_{2}\right)=\frac{2(t+\gamma) d^{\prime}(t)-d(t)}{2(t+\gamma)^{3 / 2}}, \quad t \in I .
$$

LEMma 5.1. Suppose conditions $\left(Q_{1}\right)$ and $\left(Q_{\alpha, \beta}\right)$ hold with $0<\alpha+\beta \leq \pi / 2$. (i) If $s_{1}>s_{2} \geq 0$ and $S_{1}=S_{2} \geq 0$ then $D_{\rho}(t)<0$ for all $t \in I$. (ii) If $s_{1}=s_{2} \geq 0$ and $S_{1}>S_{2} \geq 0$ then $D_{\rho}(t)>0$ for all $t \in I$, provided that $\alpha, \beta$ are additionally restricted by (4.3) if $D(x) \geq 0$ is violated.

Proof. While (i) is an immediate consequence of Lemma 4.1, a proof of assertion (ii) is more involved. If $D(x) \geq 0$ holds, we refer to [7, p. 788]. Otherwise, we define $w_{k}(t):=\sin (\alpha t+\beta / k), k \geq 1$, and consider a new variable $v:=d / w_{k}$. Notice that $v$ solves the equation (4.4) with $w(t)$ replaced by $w_{k}(t)$. Since $v(0)=0$ and $v(1)>0$, an argument as in the proof of Lemma 4.1(c) shows that $v^{\prime}(t)>0, t \in I$. Now,

$$
D_{\rho}(t)=\frac{w_{k}^{\prime}(t)}{2 \alpha(t+\gamma)^{3 / 2}} c(t) v(t)+\frac{w_{k}(t)}{\sqrt{t+\gamma}} v^{\prime}(t), \quad t \in I,
$$

where the function $c(t):=2 \alpha(t+\gamma)-\tan (\alpha t+\beta / k)$ is concave. Choosing $k \geq 1$ sufficiently large such that $c(0) \geq 0$ holds, we obtain $c(t) \geq 0$ and therefore $D_{\rho}(t)>$ $0, t \in I$, since $c(1)>0$ is valid by condition (4.3).

From Lemma 4.2 it follows that the mapping $(s, S) \mapsto \rho_{t}(s, S)$ is uniformly continuous with respect to $t \in I$ at each point $(s, S) \in E x(s, S)$. Thus, continuity is preserved by the function

$$
g(s, S):=\min _{t \in I} \rho_{t}(s, S)
$$

which is appropriate to measure positivity of $\rho(t)$. Note that the set $W(s, S):=$ $\{(s, S) \mid g(s, S) \geq 0\}$ is the natural domain of existence of wrinkle-free solutions. For an examination of its boundary the following implicit function result is needed. 
LEMMA 5.2. There exists a uniquely defined strictly increasing and continuous function $\Delta: \overline{\mathbb{R}}_{+} \rightarrow \mathbb{R}, \Delta(s)=S$, satisfying $g(s, \Delta(s))=0$ for all $s \geq 0$, provided that the assertions of Lemma 5.1 hold.

Proof. From Lemma 5.1 it follows that for each $s \geq 0$ and $S_{1}>S_{2} \geq 0$

$$
g\left(s, S_{1}\right)-g\left(s, S_{2}\right) \geq \min _{t \in I}\left(\rho_{t}\left(s, S_{1}\right)-\rho_{t}\left(s, S_{2}\right)\right)>0 .
$$

Thus existence, uniqueness, and continuity of $\Delta(s)$ are an immediate consequence of a known implicit function theorem (see [6, Thm. 51]), provided the range of $g\left(s_{0}, \cdot\right)$, $s_{0} \geq 0$ fixed, contains the point 0 . In order to prove the latter, observe first that the rn-solution $x(t):=A_{t}\left(s_{0}, s_{0}\right)$ has $C^{1}(I)$-regularity. Since $x(t) \not \equiv s_{0}$, we have $x^{\prime}\left(t_{0}\right)<0$ for at least one point $t_{0} \in I$. This shows $g\left(s_{0}, s_{0}\right)<0$. Next, by virtue of (3.1) and (4.9), one has

$$
\begin{aligned}
\rho_{t}^{*}\left(s_{0}, S\right):= & 2(t+\gamma)^{3 / 2} \rho_{t}\left(s_{0}, S\right) \\
= & (t+2 \gamma)\left(S-\int_{t}^{1}(1-\tau) F(\tau, x(\tau)) d \tau\right) \\
& -(t+2 \gamma+1)\left(s_{0}-\int_{0}^{t} \tau F(\tau, x(\tau)) d \tau\right) .
\end{aligned}
$$

Using Lebesgue's dominated convergence theorem as in the proof of Lemma 4.3, it follows that $\rho_{t}^{*}\left(s_{0}, S\right) \rightarrow+\infty$, uniformly with respect to $t \in I$ as $S \rightarrow+\infty$. Thus, the range of $g\left(s_{0}, \cdot\right)$ encloses a segment $(-\delta,+\infty)$ for some $\delta=\delta\left(s_{0}\right)>0$.

In order to prove strict monotonicity of $\Delta(s)$, let $s_{1}>s_{2} \geq 0$ be fixed. An assumption $\Delta\left(s_{1}\right) \leq \Delta\left(s_{2}\right)$ is contradictory because of

$$
\begin{aligned}
\min _{t \in I} \rho_{t}\left(s_{1}, \Delta\left(s_{2}\right)\right) & <\min _{t \in I} \rho_{t}\left(s_{2}, \Delta\left(s_{2}\right)\right)=g\left(s_{2}, \Delta\left(s_{2}\right)\right)=0=g\left(s_{1}, \Delta\left(s_{1}\right)\right) \\
& =\min _{t \in I} \rho_{t}\left(s_{1}, \Delta\left(s_{1}\right)\right) \leq \min _{t \in I} \rho_{t}\left(s_{1}, \Delta\left(s_{2}\right)\right) .
\end{aligned}
$$

Here, Lemma 5.1 was used. Thus, $\Delta\left(s_{1}\right)>\Delta\left(s_{2}\right)$ and the lemma is proved.

Due to monotonicity, we have $g(s, S)>0$ for each fixed $s \geq 0$ and $S>\Delta(s)$. Therefore, the finite part of the boundary of $W(s, S)$ is the curve $\Delta(s)$, and the following theorem needs no proof.

Theorem 5.3. Suppose conditions $(\mathrm{Q} 1)$ and $\left(\mathrm{Q}_{\alpha, \beta}\right)$ hold, with $0<\alpha+\beta \leq \pi / 2$ being restricted by (4.3) if $D(x) \geq 0$ is violated. Then the domain of existence and uniqueness of wrinkle-free solutions of the BVP $(s, S)$ is the set

$$
W(s, S)=\{(s, S) \mid s \geq 0, S \geq \Delta(s)\} .
$$

For fixed $s \geq 0$ and $S>\Delta(s)$, the circumferential stress $S_{\theta}$ is strictly positive everywhere in the membrane while for $S=\Delta(s)$ there exists at least one point where $S_{\theta}$ vanishes.

In an earlier paper $\mathrm{H}$. Grabmüller [7] has studied wrinkle-free solutions in the special case of a flat annular membrane upon the basis of the simplified Reissner model. He proved that a maximum principle holds for the circumferential stress such that the minimum of $S_{\theta}(\sigma)$ is attained at one of the edges $\sigma=0$ or $\sigma=L$. 
In the present approach, an analytic determination of the point $t \in I$ where the minimum of the function (5.3) is attained, seems to be impossible. However, as we will prove below, the relation $g(s, S)=\rho_{0}(s, S)$ holds for sufficiently large $s>0$ and $S=\Delta(s)$. Thus, the minimum is attained at the inner edge of the annulus.

A first step of a proof consists in showing that $\rho_{0}(0, S)>0$ is valid for all $S \geq 0$. Indeed, we have $F(t, x(t))-\widetilde{F}(t) x(t) \leq 0$ by condition (Q1), where $x$ denotes the rn-solution $x(t)=A_{t}(0, S), S \geq 0$, and where $\widetilde{F}(t)$ was defined in Sec. 3. Thus, the function $z(t):=x(t) / \sqrt{t+\gamma}$ satisfies the differential inequality

$$
(-z)^{\prime \prime}+\frac{1}{t+\gamma}(-z)^{\prime}-\left(\frac{1}{4(t+\gamma)^{2}}+\widetilde{F}(t)\right)(-z) \geq 0, \quad t \in I_{0} .
$$

Using an argument similar to the proof of Lemma 4.1(c), it follows that the above inequality is subordinate to the maximum principle (see [15, Theorem 4]), and therefore $z^{\prime}(0)=\rho_{0}(0, S)>0$ holds.

Next, we note the following representation of the mapping $\rho_{0}: \overline{\mathbb{R}}_{+} \times \overline{\mathbb{R}}_{+} \rightarrow \mathbb{R}$ :

$$
\rho_{0}(s, S)=\frac{1}{\sqrt{\gamma}}\left(S-\int_{0}^{1}(1-\tau) F(\tau, x(\tau)) d \tau\right)-\frac{2 \gamma+1}{2 \gamma^{3 / 2}} s .
$$

For fixed $S \geq 0$, we have $\rho_{0}(0, S)>0$ as shown above and $\rho_{0}(s, S) \rightarrow-\infty$ as $s \rightarrow+\infty$. Since the mapping $s \mapsto \rho_{0}(s, S)$ is strictly decreasing, an implicit function argument applies.

Lemma 5.4. Suppose conditions $(\mathrm{Q} 1)$ and $\left(\mathrm{Q}_{\alpha, \beta}\right)$ hold, with $0<\alpha+\beta \leq \pi / 2$ being restricted by (4.3) if $D(x) \geq 0$ is violated. Then there exists a uniquely defined strictly increasing and continuous function $\Delta_{0}^{-1}$ satisfying $\rho_{0}\left(\Delta_{0}^{-1}(S), S\right)=0$ for all $S \geq 0$, which maps the set $\overline{\mathbb{R}}_{+}$homeomorphically onto the segment $\left[s^{*},+\infty\right)$, $s^{*}:=\Delta_{0}^{-1}(0)>0$.

Proof. It remains to show that $\Delta_{0}^{-1}$ is strictly increasing with $\Delta_{0}^{-1}(S) \rightarrow+\infty$ as $S \rightarrow+\infty$. In fact, for fixed $S_{1}>S_{2} \geq 0$ an assumption $\Delta_{0}^{-1}\left(S_{1}\right) \leq \Delta_{0}^{-1}\left(S_{2}\right)$ is contradictory to the assertions of Lemma 5.1. Furthermore, from $\rho_{0}\left(\Delta_{0}^{-1}(S), S\right)=0$ and from (5.8) it follows that

$$
\Delta_{0}^{-1}(S)=\frac{2 \gamma}{2 \gamma+1} S+O(S) \rightarrow+\infty \text { as } S \rightarrow+\infty .
$$

In order to complete the proof, observe that $\rho_{0}(0,0)>0$ implies $\Delta_{0}^{-1}(0)>0$.

Now we are prepared to show that the curves $\Delta_{0}:\left[s^{*},+\infty\right) \rightarrow[0,+\infty)$ and $\Delta(s)$ coincide for sufficiently large $s \geq s^{*}$. A proof makes use of the representation

$$
\Delta_{0}(s)=\frac{2 \gamma+1}{2 \gamma} s+\int_{0}^{1}(1-\tau) F(\tau, x(\tau)) d \tau, \quad s \geq s^{*},
$$

which follows from (5.8). Observe that the defining equation $g(s, \Delta(s))=0$ of the curve $\Delta(s)$ can also be written as $\min _{t \in I} \rho_{t}^{*}(s, \Delta(s))=0$. Calculating $(d / d t) \rho_{t}^{*}(s, S)$ from (5.6) and using (5.10), one obtains

$$
\frac{d}{d t} \rho_{t}^{*}\left(s, \Delta_{0}(s)\right)=\frac{s}{2 \gamma}+\int_{0}^{t} F(\tau, x(\tau)) d \tau+2(t+\gamma) F(t, x(t)) \geq \frac{s}{2 \gamma}-(3+2 \gamma) K_{1}, \quad t \in I_{0} .
$$


We see that for $s \geq s_{k}:=2 \gamma(3+2 \gamma) K_{1}$,

$$
\min _{t \in I} \rho_{t}^{*}\left(s, \Delta_{0}(s)\right)=\rho_{0}^{*}\left(s, \Delta_{0}(s)\right)=2 \gamma^{3 / 2} \rho_{0}\left(s, \Delta_{0}(s)\right)=0,
$$

and therefore, $\Delta(s)=\Delta_{0}(s)$. This result leads via (5.10) to the following asymptotic form of the boundary arc $\Delta(s)$ :

LEMMA 5.5. $\varepsilon^{2} \Delta(s)-\left(1-\frac{\omega}{2}\right) s+K_{0, \infty}=o(1) \quad$ as $s \rightarrow+\infty$.

5.2. Wrinkle-free solutions of the displacement problems. Using a mapping argument as in Sec. 4, it becomes obvious that the subdomain $W(s, S) \subset \operatorname{Ex}(s, S)$ of wrinkle-free solutions can be mapped onto a corresponding subdomain in the boundary-parameter ranges of the BVPs $(h, S),(s, H)$, and $(h, H)$. Of prior interest is the behaviour of the boundary curve $\Delta(s)$ under the mappings (4.1) and (4.2). We notice that, by Lemma 5.2, the mapping $\Delta(s)$ constitutes a homeomorphism from the set $\overline{\mathbb{R}}_{+}$onto the segment $\left[S^{*},+\infty\right)$, where $S^{*}:=\Delta(0)$. We have $S^{*}>0$ because of $g(0,0)<0$. Thus, the inverse mapping $\Delta^{-1}:\left[S^{*},+\infty\right) \rightarrow[0,+\infty)$ exists as a strictly increasing continuous function. Now, the continuous curves

$$
\begin{aligned}
& h(S):=\varepsilon^{2} D A_{0}\left(\Delta^{-1}(S), S\right)-\frac{\omega}{2}\left(\Delta^{-1}(S)+\nu \sqrt{\left(\Delta^{-1}(S)\right)^{2}+\varepsilon^{2} y_{0}^{2}}\right), S \geq S^{*}, \\
& H(s):=D A_{1}(s, \Delta(s))-\frac{\omega}{2}\left(\Delta(s)+\nu \sqrt{\Delta^{2}(s)+y_{1}^{2}}\right), \quad s \geq 0,
\end{aligned}
$$

are properly defined and they characterize the subsets of wrinkle-free solutions of the BVPs $(h, S)$ and $(s, H)$ as follows.

THEOREM 5.6. Suppose the hypotheses of Thm. 4.5 hold. Then we have the following.

(a) The domain of existence and uniqueness of wrinkle-free solutions of the BVP $(h, S)$ is the set

$$
W(h, S):=\left\{(h, S) \mid \Gamma_{0}(S) \geq h \geq h(S), S \geq S^{*}\right\} \subset \operatorname{Ex}(h, S) .
$$

For $S \geq S_{k}:=\Delta\left(s_{k}\right)$, the representation of the boundary curve $h(S)$ simplifies to

$$
h(S)=-\frac{\omega}{2} \nu \sqrt{\left(\Delta^{-1}(S)\right)^{2}+\varepsilon^{2} y_{0}^{2}},
$$

and therefore an asymptotic form of $h(S)$ is provided by $h(S)+b_{0}\left(S+K_{0, \infty} / \varepsilon^{2}\right)=$ $o(1)$ as $S \rightarrow+\infty$, letting $b_{0}:=\varepsilon^{2} \omega \nu /\left(1+\varepsilon^{2}\right)$.

(b) The domain of existence and uniqueness of wrinkle-free solutions of the BVP $(s, H)$ is the set

$$
W(s, H):=\{(s, H) \mid s \geq 0, H \geq H(s)\} \subset \operatorname{Ex}(s, H) .
$$

For $s \geq s_{k}$, the function $\Delta(s)$ defining the boundary curve $H(s)$ may be replaced by $\Delta_{0}(s)$ and therefore an asymptotic form of $H(s)$ is provided by $H(s)-a_{0} s+$ $(1-\mu) K_{0, \infty} / \varepsilon^{2}-K_{1, \infty}=o(1)$ as $s \rightarrow+\infty$, letting $a_{0}:=\omega\left[1-\frac{1}{2}\left(1+\varepsilon^{2}\right)(1+\nu)\right]$ $/\left(2 \varepsilon^{2}\right)$.

It remains to consider the BVP $(h, H)$. In the case of $\nu=0$, the boundary of the subdomain of wrinkle-free solutions partially coincides with the semi-axis $h=0$. In 
the case of $\nu>0$, the boundary curve is most appropriately represented in parameter form $h=h(s), H=H(s), s \geq 0$, where

$$
h(s):=\varepsilon^{2} D A_{0}(s, \Delta(s))-\frac{\omega}{2}\left(s+\nu \sqrt{s^{2}+\varepsilon^{2} y_{0}^{2}}\right), \quad s \geq 0 .
$$

Theorem 5.7. Suppose the hypotheses of Thm. 4.7 hold. Then the domain of existence and uniqueness of wrinkle-free solutions of the BVP $(h, H)$ is the set

$$
W(h, H):=\{(h, H) \mid h \geq h(s), H \geq H(s), s \geq 0\} \cap \operatorname{Ex}(h, H) .
$$

For $s \geq s_{k}$, the representation of the function $h(s)$ simplifies to

$$
h(s)=-\frac{\omega}{2} \nu \sqrt{s^{2}+\varepsilon^{2} y_{0}^{2}},
$$

and therefore an asymptotic form of $H(h):=H(s(h))$ is provided by $H(h)+c_{0} h-$ $(1-\mu) K_{0, \infty} / \varepsilon^{2}+K_{1, \infty}=o(1)$ as $h \rightarrow-\infty$, letting $c_{0}:=2 a_{0} /(\omega \nu)$.

5.3. Concluding remarks. The assertions of the preceding theorems provide asymptotic forms of the wrinkling set boundaries. The slope parameters $a_{0}, b_{0}, c_{0}$ occurring in Thms. 5.6 and 5.7 are independent of the particular shape function $p(t)$ of the membrane. As previously indicated, a flat annular membrane with inner and outer radii $r=a, r=b$ is associated with a shape function $p(t)=1+\frac{a}{b}$ and thus leads us to consider $K_{0, \infty}=0=K_{1, \infty}$. Asymptotic forms of the same kind have been derived in [7] where the analysis was governed by the nonlinear membrane equations of the simplified Reissner model.

An analytic determination of the separatrices between the domains of existence and nonexistence of rn-solutions is apparently impossible. The same holds true for the subsets of wrinkle-free solutions. However, a computational calculation of the boundary curves is a standard problem of solving some boundary-value problems numerically. For example, a calculation of $\Gamma_{0}$ as defined in (4.12) requires the numerical integration of the boundary-value problem

$$
x^{\prime \prime}(t)=F(t, x(t)), \quad t \in I_{0}, x(0)=0, x(1)=S, S \geq 0 .
$$

Having determined the numerical solution $\tilde{x}_{0}(t)=\tilde{A}_{t}(0, S)$ and its first derivative, an approximation of $\Gamma_{0}$ is obtained in the form $\Gamma_{0}(S) \doteq \varepsilon^{2} \tilde{x}_{0}^{\prime}(0)-\frac{\omega}{2} \nu \varepsilon\left|y_{0}\right|$. For sufficiently large $S \gg 0$ the asymptotic form (4.8) can be utilized.

Some computational results are documented in Figs. 1 to 4 where a uniform surface load $q_{\zeta}=q_{0}=1$ and an aperture $a=0.1$ are assumed and where we let $C=0.25$, $\nu=0.33$, and $L=1$. The following examples are considered for a restricted arclength $0 \leq \sigma \leq 1$ :

$$
\begin{array}{llll}
r(\sigma):=a+\sigma, & y(\sigma)=y_{0}-\left(a \sigma+\frac{1}{2} \sigma^{2}\right), & y_{0}:=0, & \left(E_{1}\right) \\
r(\sigma):=a+\sigma, & y(\sigma)=y_{0}-\left(a \sigma+\frac{1}{2} \sigma^{2}\right), & y_{0}:=1, & \left(E_{2}\right) \\
r(\sigma):=a+\frac{4}{\pi} \sin \frac{\pi \sigma}{4}, & y(\sigma)=y_{0}-\left(a \sigma+\left(\frac{4}{\pi}\right)^{2}\left(1-\cos \frac{\pi \sigma}{4}\right)\right), y_{0}:=1, & \left(E_{3}\right) \\
r(\sigma):=a+\frac{2}{\pi} \sin \frac{\pi \sigma}{2}, & y(\sigma)=y_{0}-\left(a \sigma+\left(\frac{2}{\pi}\right)^{2}\left(1-\cos \frac{\pi \sigma}{2}\right)\right), y_{0}:=1 . & & \left(E_{4}\right)
\end{array}
$$




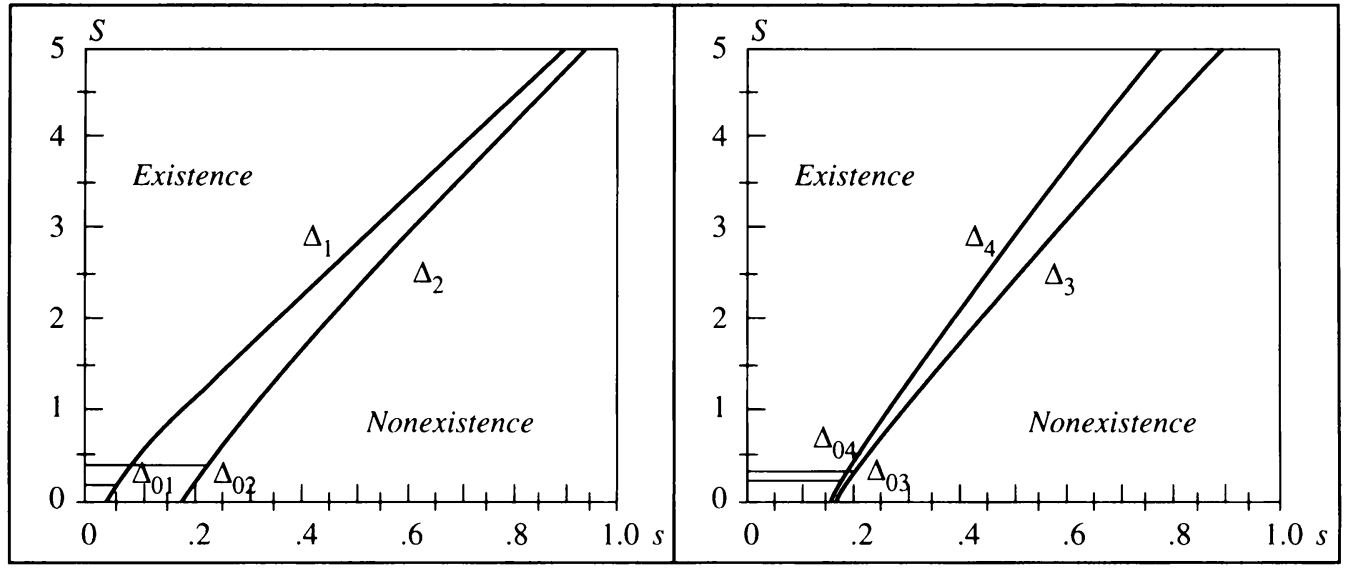

FIG. 1. The domains of wrinkle-free solutions of the BVP $(s, S)$ for flat (left) and curved (right) annular membranes. The load is uniform and Poisson's ratio is $\nu=0.33$.

The first two examples describe a flat annular membrane of outer radius $b=1.1$ having a shape function $p(t)=1+\varepsilon$ with $\varepsilon=1 / 11$. Examples $\left(\mathrm{E}_{3}\right)$ and $\left(\mathrm{E}_{4}\right)$ are curved annular membranes (spherical caps) of outer radii $b=0.1+2 \sqrt{2} / \pi$ and $b=0.1+2 / \pi$, respectively. An analytic determination of the parameter $\varepsilon$ and the shape function $p(t)$ is possible but rather tedious. We restrict ourselves to a communication of the numerical values $\varepsilon \doteq 0.07655$ (example $\left(\mathrm{E}_{3}\right)$ ) and $\varepsilon \doteq$ 0.08747 (example $\left.\left(\mathrm{E}_{4}\right)\right)$. The condition $y \geq 0$ apparently holds for $\left(\mathrm{E}_{2}\right)-\left(\mathrm{E}_{4}\right)$ and therefore, all requirements of the preceding analysis are fulfilled. Concerning example $\left(E_{1}\right)$, it is easily checked that condition $(Q 1)$ is valid while $\left(Q_{\alpha, \beta}\right)$ fails to hold for any $\alpha \geq 0$. Nevertheless, numerical computations show that positive solutions do exist admitting the correct determination of the separatrices as in the other examples.

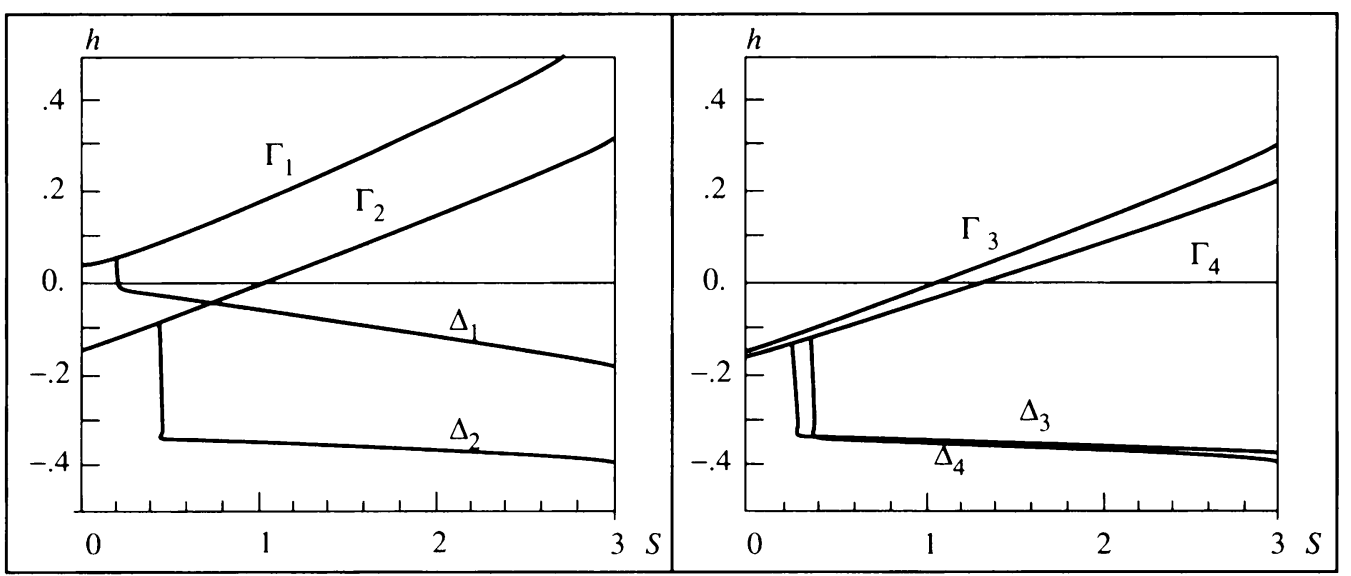

FIG. 2. The domains of wrinkle-free solutions of the BVP $(h, S)$ 


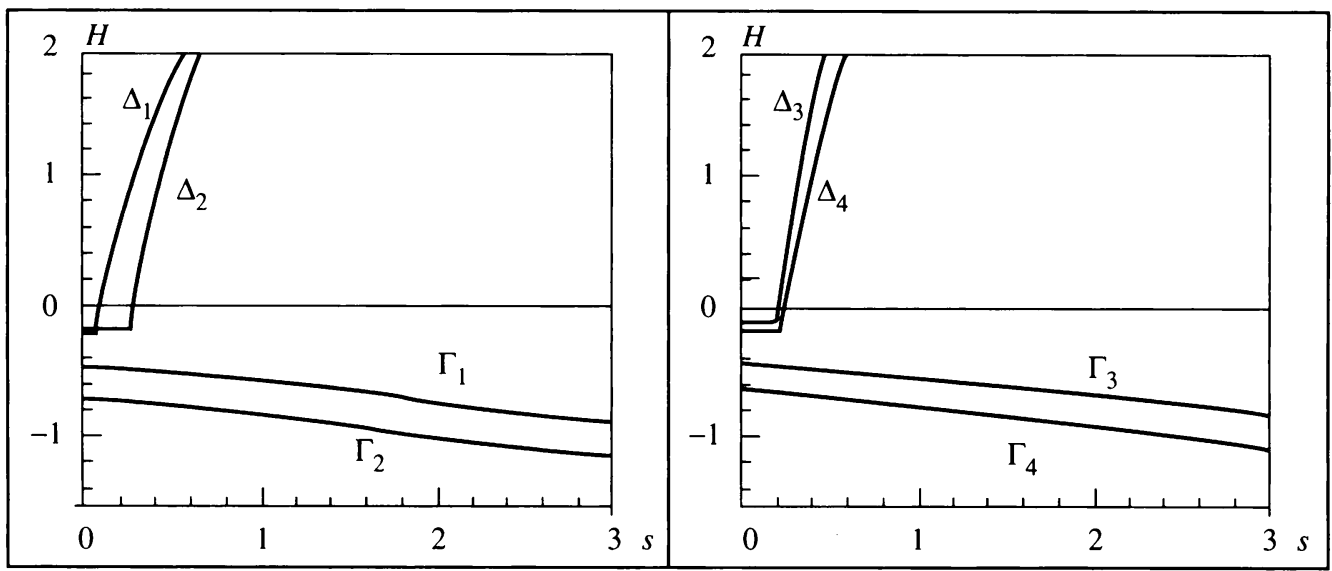

FIG. 3. The domains of wrinkle-free solutions of the BVP $(s, H)$

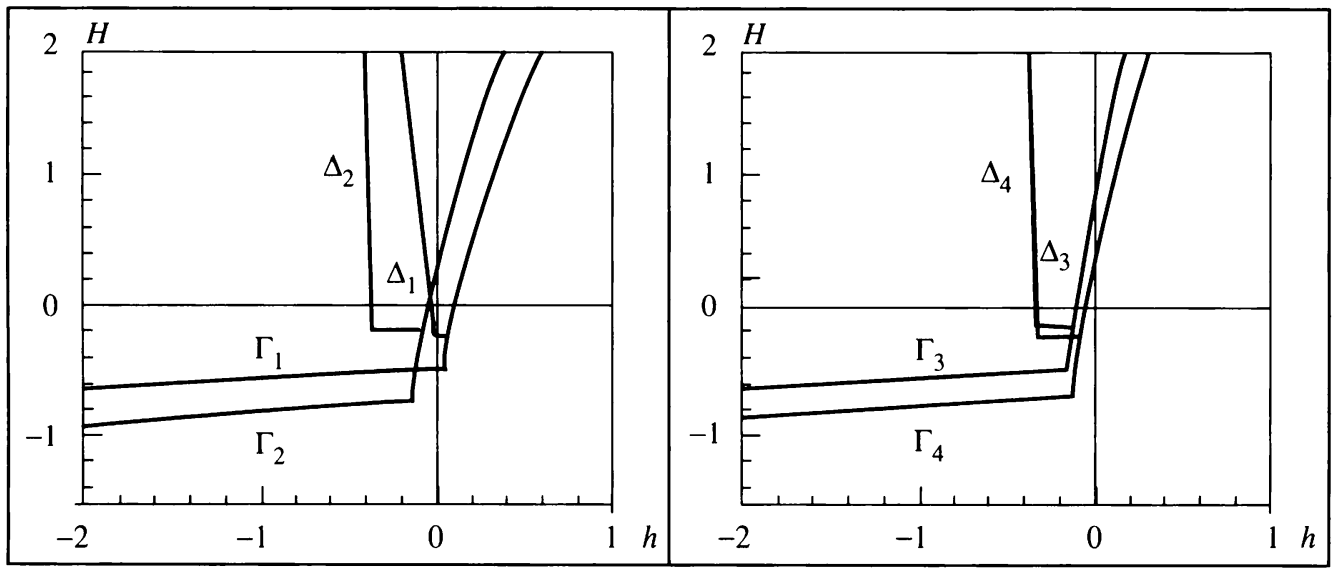

FIG. 4. The domains of wrinkle-free solutions of the BVP $(h, H)$

Notations are as follows. Separatrices between the domains of existence and nonexistence of rn-solutions are denoted by $\Gamma_{i}$ while wrinkling set boundaries are denoted by $\Delta_{i}$. An index $i$ corresponds to example $\left(\mathrm{E}_{i}\right)$. The curves $\Delta_{0 i}$ are taken from (5.10).

\section{REFERENCES}

[1] J. Arango, Existenz und Eindeutigkeit über Lösungen von Randwertproblemen der nichtlinearen Membrantheorie, Dissertation, Faculty of Science I, Univ. Erlangen-Nürnberg, July, 1989, 121 pp

[2] A. Beck, Nichtflache Kreis- und Kreisringmembranen unter axialsymmetrischer Vertikallast: Existenz und Eindeutigkeitsbereiche positiver Hauptspannungen und deren numerische Berechnung, Dissertation, Faculty of Science I, Univ. Erlangen-Nürnberg, July, 1992, 79 pp

[3] A. Beck and H. Grabmüller, Wrinkle-free solutions in the theory of curved circular membrane problems, J. Engineering Math. 27, 389-409 (1993) 
[4] A. J. Callegari and E. L. Reiss, Nonlinear boundary-value problems for the circular membrane, Arch. Rat. Mech. Anal. 31, 390-400 (1968)

[5] R. A. Clark and O. S. Narayanaswamy, Nonlinear membrane problems for elastic shells of revolution, Proc. Sympos. Theory of Shells (L. H. Donnell Anniversary Volume; D. Muster, ed.), Univ. of Houston Press, Houston, 1967, pp. 81-110

[6] F. Erwe, Differential- und Integralrechnung I, Mannheim, Bibliographisches Institut, 1962

[7] $\mathrm{H}$. Grabmüller, Wrinkle-free solutions in the theory of annular elastic membranes, J. Appl. Math. and Phys. (ZAMP) 42, 783-805 (1991)

[8] H. Grabmüller and E. Novak, Nonlinear boundary value problems for the annular membrane: A note on uniqueness of positive solutions, J. Elasticity 17, 279-284 (1987)

[9] H. Grabmüller and E. Novak, Nonlinear boundary value problems for the annular membrane: New results on existence of positive solutions, Math. Methods Appl. Sci. 10, 37-49 (1988)

[10] H. Grabmüller and R. Pirner, Positive solutions of annular elastic membrane problems with finite rotations, Studies in Applied Math. 77, 223-252 (1987)

[11] H. Grabmüller and R. Pirner, Existence theorems for some boundary value problems in the nonlinear theory of elastic membranes, European J. Appl. Math. 3, 299-317 (1992)

[12] H. Grabmüller and H. J. Weinitschke, Finite displacements of annular elastic membranes, J. Elasticity 16, 135-147 (1986)

[13] A. Libai and J. G. Simmonds, The nonlinear theory of elastic shells, One spatial dimension, Academic Press, Boston, 1988

[14] R. Pirner, Randwertprobleme der nichtlinearen Membrantheorie: Über Existenz und Nicht-Existenz postiver Lösungen, Diploma-Thesis, Faculty of Science I, Univ. Erlangen-Nürnberg, April, 1987, $82 \mathrm{pp}$

[15] M. H. Protter and H. F. Weinberger, Maximum principles in differential equations, Prentice Hall, Inc., Englewood Cliffs, N.J., 1967

[16] E. Reissner, On axisymmetrical deformation of thin shells of revolution, Proc. Sympos. Appl. Math., Vol. III, Amer. Math. Soc., Providence, RI, 1950, pp. 27-52

[17] E. Schwerin, Über Spannungen und Formänderungen kreisringförmiger Membranen, Z. Tech. Phys. 12, 651-659 (1929)

[18] J. G. Simmonds and A. Libai, A simplified version of Reissner's nonlinear equations for a firstapproximation theory of shells of revolution, Computational Mechanics 2, 99-103 (1987)

[19] D. J. Steigmann, Proof of a conjecture in elastic membrane theory, ASME J. Appl. Mech. 53, 955-956 (1986)

[20] W. Walter, Gewöhnliche Differentialgleichungen, Springer-Verlag, Berlin-Heidelberg-New YorkTokyo, 1986

[21] H. J. Weinitschke, Stable and unstable axisymmetric solutions for membranes of revolution, Appl. Mech. Rev. 42, No. 11, Part 2, S289-S294 (1989)

[22] H. J. Weinitschke and H. Grabmüller, Recent mathematical results in the nonlinear theory of flat and curved elastic membranes of revolution, J. Engineering Math. 26, 159-194 (1992) 